\title{
VALUES OF NONCOMMUTATIVE POLYNOMIALS, LIE SKEW-IDEALS AND TRACIAL NULLSTELLENSÄTZE
}

\author{
MATEJ BRešAR ${ }^{1}$ AND IgOR KLEP ${ }^{2}$
}

\begin{abstract}
A subspace of an algebra with involution is called a Lie skew-ideal if it is closed under Lie products with skew-symmetric elements. Lie skew-ideals are classified in central simple algebras with involution (there are eight of them for involutions of the first kind and four for involutions of the second kind) and this classification result is used to characterize noncommutative polynomials via their values in these algebras. As an application, we deduce that a polynomial is a sum of commutators and a polynomial identity of $d \times d$ matrices if and only if all of its values in the algebra of $d \times d$ matrices have zero trace.
\end{abstract}

\section{Introduction}

Interest in positivity questions of noncommutative polynomials has been recently revived by Helton's seminal paper [Hel], in which he proved that a polynomial is a sum of squares of polynomials if and only if its values in matrices of any size are positive semidefinite. A nice survey of recent functional analytic results in this direction and their various applications is given in dOHMP.

One of the results in this vein was obtained by the second author and Schweighofer. They showed that Connes' embedding conjecture on type $\mathrm{II}_{1}$ von Neumann algebras is equivalent to a problem of describing polynomials whose values at tuples of self-adjoint $d \times d$ matrices (of norm at most 1 ) have nonnegative trace for every $d \geq 1$; see $[\mathrm{KS}$, Theorem 1.6] for a precise formulation. The natural first step in understanding this problem is examining the zero trace situation. The authors proved that a polynomial whose values always have trace zero is a sum of commutators [KS, Theorem 2.1]. This result was the initial motivation for the present work.

A non-dimensionfree approach to Connes' embedding conjecture entails studying values of polynomials when evaluated at tuples of $d \times d$ matrices for a fixed $d$. A result in this spirit - a weak version of Helton's sum of squares theorem - can be obtained from the Procesi and Schacher 1976 paper [PS. A more recent reference is $\underline{\mathrm{KU}}$, where a Positivstellensatz characterizing polynomials whose values in $d \times d$ matrices are all positive semidefinite is given. The next step is to study the nonnegativity of the trace, and as a special case the zero trace. The work on this paper begun by addressing the latter problem. The solution, which we call the "tracial Nullstellensatz", is simple: a polynomial has zero trace when evaluated at $d \times d$ matrices if and only if it is a

Received by the editors October 9, 2008.

2000 Mathematics Subject Classification. Primary 16R50, Secondary 16W10.

Key words and phrases. noncommutative polynomial, Lie ideal, Lie skew-ideal, sum of commutators, trace, involution.

${ }^{1}$ Supported by the Slovenian Research Agency (program No. P1-0288).

${ }^{2}$ Supported by the Slovenian Research Agency (project No. Z1-9570-0101-06). 
sum of commutators and a polynomial identity of $d \times d$ matrices (Corollaries 4.7 and 4.12 .

The zero trace problem has led us to consider the following more general topic: What is the linear span of all the values of a polynomial on a given algebra $\mathcal{A}$ ? Studying this question has turned out to be quite fruitful. As we shall see, its answer yields tracial Nullstellensätze, and on the other hand, we believe, admittedly somewhat speculatively, that it is a natural question related to various other areas. As a matter of fact, its consideration is, as we shall see, connected to certain Lie structure topics and also to the notion of polynomial identities.

Our crucial observation is that the linear span of values of a polynomial is a Lie ideal of the algebra $\mathcal{A}$ in question (Theorem 2.3). This paves the way for the precise description. For example, in the special case where $\mathcal{A}=M_{d}(\mathbb{F})$ is the algebra of all $d \times d$ matrices over a field $\mathbb{F}$ with $\operatorname{char}(\mathbb{F})=0$, Theorem 4.5 implies that polynomials $f$ can be categorized into four classes according to their values:

(i) $f$ is a polynomial identity; in this case the span of its values is 0 ;

(ii) $f$ is a central polynomial; in this case the span of its values are the scalar matrices;

(iii) $f$ is a sum of commutators and a polynomial identity (but is not a polynomial identity); in this case the span of its values is the set of all trace zero matrices;

(iv) if $f$ is none of the above, then the span of its values is the entire algebra $M_{d}(\mathbb{F})$.

Theorem 4.5 works at a greater level of generality - it is proved for finite dimensional central simple algebras (and a version even holds for prime PI algebras, as can be easily shown).

Our main results, however, deal with algebras with involution. These theorems are of the same flavor as those outlined in the preceding paragraph, but somewhat more involved. We consider noncommutative polynomials in $X_{i}$ and $X_{i}^{*}$ (i.e., elements of a free $*$-algebra), and observe that the linear span of values of such a polynomial need not be a Lie ideal, but it is always closed under Lie products with skew-symmetric elements (Theorem 2.5). We call subspaces having this property Lie skew-ideals and classify them for finite dimensional central simple algebras (Theorems 3.13 and 3.16). Again, this enables us to categorize polynomials into classes, eight of them for an involution of the first kind and four of them for an involution of the second kind (Theorems 4.9 and 4.10 .

The tracial Nullstellensätze mentioned above are deduced easily from these results, cf. cases (i) and (iii) above. We revisit and reinterpret them in the setting of algebras of generic matrices [Pro1, Chapter III] in the last section: an element of such an algebra is a sum of commutators if and only if its trace is zero (recall that the algebra of generic matrices is a subalgebra of a matrix algebra over a polynomial algebra and thus naturally equipped with a trace).

\section{The Lie Structure of Polynomial Values}

Let us fix the notation that will be used throughout the paper. By $\mathbb{F}$ we denote a field, and all our algebras will be algebras over $\mathbb{F}$. Let $\mathcal{A}$ be an (associative) algebra. By $\mathcal{Z}$ we denote its center. If $\mathcal{A}$ is a $*$-algebra, i.e., an algebra with involution $*$, then by $\mathcal{S}$ (resp. $\mathcal{K}$ ) we denote the set of all symmetric (resp. skew-symmetric) elements in 
$\mathcal{A}:$

$$
\mathcal{S}=\left\{a \in \mathcal{A} \mid a^{*}=a\right\}, \quad \mathcal{K}=\left\{a \in \mathcal{A} \mid a^{*}=-a\right\} .
$$

The advantage of this notation is brevity, but the reader should be warned against possible confusion. Let us point out that $\mathcal{S}$ and $\mathcal{K}$ depend on the involution; we will have the opportunity to consider different involutions on the same algebra $\mathcal{A}$ (cf. Lemmas 3.10 and 3.12 , so $\mathcal{S}$ and $\mathcal{K}$ might differ from case to case.

2.1. The involution-free case. By $\mathbb{F}\langle\bar{X}\rangle$ we denote the free algebra generated by $\bar{X}=\left\{X_{1}, X_{2}, \ldots\right\}$, i.e., the algebra of all polynomials in noncommuting variables $X_{i}$. Let $\mathcal{A}$ be an algebra over $\mathbb{F}$, and let $f=f\left(X_{1}, \ldots, X_{n}\right) \in \mathbb{F}\langle\bar{X}\rangle$. If $\mathcal{L}_{1}, \ldots, \mathcal{L}_{n}$ are subsets of $\mathcal{A}$, then by $f\left(\mathcal{L}_{1}, \ldots, \mathcal{L}_{n}\right)$ we denote the set of all values $f\left(a_{1}, \ldots, a_{n}\right)$ with $a_{i} \in \mathcal{L}_{i}, i=1, \ldots, n$. If all $\mathcal{L}_{i}$ are equal to $\mathcal{A}$, then we simplify the notation and write $f(\mathcal{A})$ instead of $f(\mathcal{A}, \ldots, \mathcal{A})$. If $\mathcal{U}$ is a subset of $\mathcal{A}$, then by span $\mathcal{U}$ we denote the linear span of $\mathcal{U}$. One of the goals of this paper is to describe span $f(\mathcal{A})$ for all polynomials $f$ and certain algebras $\mathcal{A}$. Of course it can happen that $\operatorname{span} f(\mathcal{A})=0$ even when $f \neq 0$; such a polynomial $f$ is called a (polynomial) identity of $\mathcal{A}$. Algebras satisfying (nontrivial) polynomial identities are called PI algebras. This class of algebras includes all finite dimensional algebras.

We say that a polynomial $f=f\left(X_{1}, \ldots, X_{n}\right) \in \mathbb{F}\langle\bar{X}\rangle$ is homogeneous in $X_{i}$ if each monomial of $f$ has the same degree with respect to $X_{i}$; if this degree is 1 , then we say that $f$ is linear in $X_{i}$. Further, we say that $f$ is multihomogeneous if it is homogeneous in every $X_{i}, i=1, \ldots, n$. Every polynomial is a sum of multihomogeneous polynomials. A polynomial is said to be multilinear if it is linear in every $X_{i}$, $i=1, \ldots, n$. Thus, a multilinear polynomial in $X_{1}, \ldots, X_{n}$ is a linear combination of monomials of the form $X_{\sigma(1)} \ldots X_{\sigma(n)}$ where $\sigma$ is a permutation of $\{1, \ldots, n\}$. From the identity

$$
\begin{gathered}
{\left[X_{\sigma(1)} \ldots X_{\sigma(n)}, X_{n+1}\right]=\left[X_{\sigma(1)}, X_{n+1}\right] X_{\sigma(2)} \ldots X_{\sigma(n)}} \\
+X_{\sigma(1)}\left[X_{\sigma(2)}, X_{n+1}\right] X_{\sigma(3)} \ldots X_{\sigma(n)}+\ldots+X_{\sigma(1)} \ldots X_{\sigma(n-1)}\left[X_{\sigma(n)}, X_{n+1}\right]
\end{gathered}
$$

it follows easily that every multilinear polynomial $h$ satisfies (cf. [BCM, p. 170])

$$
\begin{aligned}
& {\left[h\left(X_{1}, \ldots, X_{n}\right), X_{n+1}\right]=h\left(\left[X_{1}, X_{n+1}\right], X_{2}, \ldots, X_{n}\right)} \\
& +h\left(X_{1},\left[X_{2}, X_{n+1}\right], X_{3}, \ldots, X_{n}\right)+\ldots+h\left(X_{1}, \ldots, X_{n-1},\left[X_{n}, X_{n+1}\right]\right) .
\end{aligned}
$$

In order to state our first theorem we have to recall a definition and record an elementary lemma which will be used frequently in the sequel.

Definition 2.1. An algebra $\mathcal{A}$ endowed with the Lie product

$$
[x, y]:=x y-y x \quad \text { for } x, y \in \mathcal{A}
$$

is a Lie algebra and the ideals of $\mathcal{A}$ with respect to this product are called Lie ideals of $\mathcal{A}$.

Thus, a Lie ideal of $\mathcal{A}$ is a linear subspace $\mathcal{L}$ of $\mathcal{A}$ such that $[\mathcal{L}, \mathcal{A}] \subseteq \mathcal{L}$.

Lemma 2.2. Let $\mathcal{V}$ be a linear space over $\mathbb{F}$, and let $\mathcal{U}$ be a subspace. Suppose that $c_{0}, c_{1}, \ldots, c_{n} \in \mathcal{V}$ are such that

$$
\sum_{i=0}^{n} \lambda^{i} c_{i} \in \mathcal{U}
$$


holds for at least $n+1$ different scalars $\lambda$. Then each $c_{i} \in \mathcal{U}$.

Proof. Let $\lambda_{\ell} \in \mathbb{F}, \ell=0, \ldots, n$, be different elements in $\mathbb{F}$ satisfying (2). Then

$$
\sum_{i=0}^{n} \lambda_{\ell}^{i} \bar{c}_{i}=0
$$

in the vector space $\mathcal{V} / \mathcal{U}$, where $v \mapsto \bar{v}$ denotes the quotient mapping $\mathcal{V} \rightarrow \mathcal{V} / \mathcal{U}$. The system $(3)_{\ell=0, \ldots, n}$ can be equivalently written in matrix form as

$$
\left[\begin{array}{cccc}
1 & \lambda_{0} & \cdots & \lambda_{0}^{n} \\
\vdots & \vdots & \ddots & \vdots \\
1 & \lambda_{n} & \cdots & \lambda_{n}^{n}
\end{array}\right]\left[\begin{array}{c}
\bar{c}_{0} \\
\vdots \\
\bar{c}_{n}
\end{array}\right]=\left[\begin{array}{c}
0 \\
\vdots \\
0
\end{array}\right] .
$$

The Vandermonde matrix on the left hand side is invertible as its determinant is

$$
\prod_{0 \leq i<j \leq n}\left(\lambda_{i}-\lambda_{j}\right)
$$

Thus $\bar{c}_{i}=0$, i.e., $c_{i} \in \mathcal{U}$ for all $i$.

Theorem 2.3. Let $\mathbb{F}$ be an infinite field, let $\mathcal{A}$ be an $\mathbb{F}$-algebra, and let $\mathcal{L}_{1}, \ldots, \mathcal{L}_{n}$ be Lie ideals of $\mathcal{A}$. Then for every $f=f\left(X_{1}, \ldots, X_{n}\right) \in \mathbb{F}\langle\bar{X}\rangle, \operatorname{span} f\left(\mathcal{L}_{1}, \ldots, \mathcal{L}_{n}\right)$ is again a Lie ideal of $\mathcal{A}$.

Proof. We can write $f=f_{0}+f_{1}+\ldots+f_{m}$ where $f_{i}$ is the sum of all monomials of $f$ that have degree $i$ in $X_{1}$. Note that

$$
f\left(\lambda a_{1}, a_{2}, \ldots, a_{n}\right)=\sum_{i=0}^{m} \lambda^{i} f_{i}\left(a_{1}, \ldots, a_{n}\right) \in \operatorname{span} f\left(\mathcal{L}_{1}, \ldots, \mathcal{L}_{n}\right)
$$

for all $\lambda \in \mathbb{F}$ and all $a_{i} \in \mathcal{L}_{i}$, and so $f_{i}\left(a_{1}, \ldots, a_{n}\right) \in \operatorname{span} f\left(\mathcal{L}_{1}, \ldots, \mathcal{L}_{n}\right)$ by Lemma 2.2 . Repeating the same argument with respect to other variables we see that values of each of the multihomogeneous components of $f$ lie in $\operatorname{span} f\left(\mathcal{L}_{1}, \ldots, \mathcal{L}_{n}\right)$. But then there is no loss of generality in assuming that $f$ itself is multihomogeneous. Accordingly, we can write

$$
f=h\left(X_{1}, \ldots, X_{1}, X_{2}, \ldots, X_{2}, \ldots, X_{n}, \ldots, X_{n}\right)
$$

where $h \in \mathbb{F}\langle\bar{X}\rangle$ is multilinear, $X_{1}$ appears $k_{1}$ times, $X_{2}$ appears $k_{2}$ times, etc. Considering $f\left(a_{1}+\lambda a_{1}^{\prime}, a_{2}, \ldots, a_{n}\right)$ we thus arrive at the relation

$$
\sum_{i=0}^{k_{1}} \lambda^{i} c_{i} \in \operatorname{span} f\left(\mathcal{L}_{1}, \ldots, \mathcal{L}_{n}\right)
$$

where, in particular,

$$
\begin{aligned}
c_{1} & =h\left(a_{1}^{\prime}, a_{1}, \ldots, a_{1}, a_{2}, \ldots, a_{2}, \ldots, a_{n}, \ldots, a_{n}\right) \\
& +h\left(a_{1}, a_{1}^{\prime}, a_{1}, \ldots, a_{1}, a_{2}, \ldots, a_{2}, \ldots, a_{n}, \ldots, a_{n}\right) \\
& +\ldots+h\left(a_{1}, \ldots, a_{1}, a_{1}^{\prime}, a_{2}, \ldots, a_{2}, \ldots, a_{n}, \ldots, a_{n}\right) .
\end{aligned}
$$

By Lemma 2.2 each $c_{i}$, including of course $c_{1}$, belongs to $\operatorname{span} f\left(\mathcal{L}_{1}, \ldots, \mathcal{L}_{n}\right)$; here, $a_{1}, a_{1}^{\prime} \in \mathcal{L}_{1} a_{2} \in \mathcal{L}_{2}, \ldots, a_{n} \in \mathcal{L}_{n}$ are arbitrary elements. Similar statements can be established with respect to other variables. 
Now, using (1) we see that for all $a_{i} \in \mathcal{L}_{i}$ and $b \in \mathcal{A}$ we have

$$
\begin{array}{r}
{\left[f\left(a_{1}, \ldots, a_{n}\right), b\right]=h\left(\left[a_{1}, b\right], a_{1}, \ldots, a_{1}, a_{2}, \ldots, a_{2}, \ldots, a_{n}, \ldots, a_{n}\right)} \\
+\ldots+h\left(a_{1}, \ldots, a_{1},\left[a_{1}, b\right], a_{2}, \ldots, a_{2}, \ldots, a_{n}, \ldots, a_{n}\right) \\
+\ldots+h\left(a_{1}, \ldots, a_{1},\left[a_{2}, b\right], a_{2}, \ldots, a_{2}, \ldots, a_{n}, \ldots, a_{n}\right) \\
+\ldots+h\left(a_{1}, \ldots, a_{1}, a_{2}, \ldots, a_{2},\left[a_{2}, b\right], \ldots, a_{n}, \ldots, a_{n}\right) \\
+\ldots+h\left(a_{1}, \ldots, a_{1}, a_{2}, \ldots, a_{2}, \ldots,\left[a_{n}, b\right], a_{n} \ldots, a_{n}\right) \\
+\ldots+h\left(a_{1}, \ldots, a_{1}, a_{2}, \ldots, a_{2}, \ldots, a_{n} \ldots, a_{n},\left[a_{n}, b\right]\right) .
\end{array}
$$

Let us point out that $\left[a_{i}, b\right] \in \mathcal{L}_{i}$ since $\mathcal{L}_{i}$ is a Lie ideal of $\mathcal{A}$. In view of the above observation $c_{1} \in \operatorname{span} f\left(\mathcal{L}_{1}, \ldots, \mathcal{L}_{n}\right)$ it follows that the sum of the first $k_{1}$ summands that involve $\left[a_{1}, b\right]$ lies in span $f\left(\mathcal{L}_{1}, \ldots, \mathcal{L}_{n}\right)$. Similarly we see that the sum of summands involving $\left[a_{2}, b\right]$ lies in span $f\left(\mathcal{L}_{1}, \ldots, \mathcal{L}_{n}\right)$, etc. Accordingly, $\left[f\left(a_{1}, \ldots, a_{n}\right), b\right] \in$ span $f\left(\mathcal{L}_{1}, \ldots, \mathcal{L}_{n}\right)$, proving that $\operatorname{span} f\left(\mathcal{L}_{1}, \ldots, \mathcal{L}_{n}\right)$ is a Lie ideal of $\mathcal{A}$.

A very special case of Theorem 2.3 , where $f=\left[\left[X_{1}, X_{2}\right], X_{2}\right]$, was noticed in the recent paper [BKS, Lemma 4.6] as an auxiliary, but important result needed for describing Lie ideals of tensor products of algebras.

2.2. The involution case. For dealing with polynomial values in algebras with involution we introduce the analogue of a free algebra in the category of algebras with involution. Let $\mathbb{F}$ be a field with an involution $*$. By $\mathbb{F}\left\langle\bar{X}, \bar{X}^{*}\right\rangle$ we denote the free $*$ algebra over $\mathbb{F}$ generated by $\bar{X}=\left\{X_{1}, X_{2}, \ldots\right\}$, i.e., the $\mathbb{F}$-algebra of all polynomials in noncommuting variables $X_{i}, X_{j}^{*}$. Further, by $\operatorname{Sym} \mathbb{F}\left\langle\bar{X}, \bar{X}^{*}\right\rangle$ we denote the set of all symmetric, and by Skew $\mathbb{F}\left\langle\bar{X}, \bar{X}^{*}\right\rangle$ we denote the set of all skew-symmetric polynomials in $\mathbb{F}\left\langle\bar{X}, \bar{X}^{*}\right\rangle$ (with respect to the canonical involution, of course). By the degree of $X_{i}$ in a monomial $M \in \mathbb{F}\left\langle\bar{X}, \bar{X}^{*}\right\rangle$ we shall mean the number of appearances of $X_{i}$ or $X_{i}^{*}$ in $M$. For example, both $X_{1}^{2}$ and $X_{1} X_{1}^{*}$ have degree 2 in $X_{1}$. The concepts of (multi)homogeneity and (multi)linearity of polynomials in $\mathbb{F}\left\langle\bar{X}, \bar{X}{ }^{*}\right\rangle$ are defined accordingly. For example, $X_{1} X_{2} X_{1}^{*}+X_{2}^{*} X_{1}^{2}$ is multihomogeneous and linear in $X_{2}$.

Let $\mathcal{A}$ be an algebra with involution $*$ and let $f=f\left(X_{1}, \ldots, X_{n}, X_{1}^{*}, \ldots, X_{n}^{*}\right) \in$ $\mathbb{F}\left\langle\bar{X}, \bar{X}^{*}\right\rangle$. If $\mathcal{L}_{1}, \ldots, \mathcal{L}_{n}$ are subsets of $\mathcal{A}$, then by $f\left(\mathcal{L}_{1}, \ldots, \mathcal{L}_{n}\right)$ we denote the set of all values $f\left(a_{1}, \ldots, a_{n}, a_{1}^{*}, \ldots, a_{n}^{*}\right)$ with $a_{i} \in \mathcal{L}_{i}, i=1, \ldots, n$. Again, if $\mathcal{L}_{i}=\mathcal{A}$ for every $i$, then we simply write $f(\mathcal{A})$ instead of $f(\mathcal{A}, \ldots, \mathcal{A})$.

Theorem 2.3 does not hold for polynomials in $\mathbb{F}\left\langle\bar{X}, \bar{X}^{*}\right\rangle$. For example, if $f=X_{1}+$ $X_{1}^{*}$, then (assuming $\left.\operatorname{char}(\mathbb{F}) \neq 2\right) f(\mathcal{A})=\mathcal{S}$ and so $\operatorname{span} f(\mathcal{A})$ is only exceptionally a Lie ideal of $\mathcal{A}$. However, it does satisfy a weaker version of the definition of a Lie ideal: while it is, in general, not closed under commutation with elements from $\mathcal{S}$, it is certainly closed under commutation with elements from $\mathcal{K}$ since $[\mathcal{S}, \mathcal{K}] \subseteq \mathcal{S}$. Subspaces satisfying this property will be one of the central topics of this paper.

Definition 2.4. A linear subspace $\mathcal{L}$ of an algebra $\mathcal{A}$ with involution will be called a Lie skew-ideal of $\mathcal{A}$ if $[\mathcal{L}, \mathcal{K}] \subseteq \mathcal{L}$.

Theorem 2.5. Let $\mathbb{F}$ be an infinite field with char $(\mathbb{F}) \neq 2$, let $\mathcal{A}$ be an $\mathbb{F}$-algebra with involution, and let $\mathcal{L}_{1}, \ldots, \mathcal{L}_{n}$ be Lie skew-ideals of $\mathcal{A}$. Then for every $f=$ 
$f\left(X_{1}, \ldots, X_{n}, X_{1}^{*}, \ldots, X_{n}^{*}\right) \in \mathbb{F}\left\langle\bar{X}, \bar{X}^{*}\right\rangle$, span $f\left(\mathcal{L}_{1}, \ldots, \mathcal{L}_{n}\right)$ is again a Lie skew-ideal of $\mathcal{A}$.

Proof. The proof is almost the same as the proof of Theorem 2.3, so we only point out the necessary modifications.

The first part of the proof based on applications of Lemma 2.2 is literally the same, except that instead of scalars in $\mathbb{F}$ one should deal with scalars from the subfield $\mathbb{F}_{0}$ of all symmetric elements of $\mathbb{F}$. Since $\left[\mathbb{F}: \mathbb{F}_{0}\right] \leq 2, \mathbb{F}_{0}$ is also an infinite field, and so all arguments still work.

Let $h=h\left(X_{1}, \ldots, X_{n}, X_{1}^{*}, \ldots, X_{n}^{*}\right) \in \mathbb{F}\left\langle\bar{X}, \bar{X}^{*}\right\rangle$ be a multilinear polynomial. The formula (1) does not hold for $h$ (not even if $h=X_{1}^{*}$ ). However, using $\left[X_{i}^{*}, X_{n+1}-\right.$ $\left.X_{n+1}^{*}\right]=\left[X_{i}, X_{n+1}-X_{n+1}^{*}\right]^{*}$ one easily derives the following analogous formula

$$
\begin{aligned}
& {\left[h\left(X_{1}, \ldots, X_{n}, X_{1}^{*}, \ldots, X_{n}^{*}\right), X_{n+1}-X_{n+1}^{*}\right] } \\
= & h\left(\left[X_{1}, X_{n+1}-X_{n+1}^{*}\right], X_{2}, \ldots, X_{n},\left[X_{1}, X_{n+1}-X_{n+1}^{*}\right]^{*}, X_{2}^{*}, \ldots, X_{n}^{*}\right) \\
+ & h\left(X_{1},\left[X_{2}, X_{n+1}-X_{n+1}^{*}\right], X_{3}, \ldots, X_{n}, X_{1}^{*},\left[X_{2}, X_{n+1}-X_{n+1}^{*}\right]^{*}, X_{3}^{*}, \ldots, X_{n}^{*}\right) \\
+ & \ldots+h\left(X_{1}, \ldots, X_{n-1},\left[X_{n}, X_{n+1}-X_{n+1}^{*}\right], X_{1}^{*}, \ldots, X_{n-1}^{*},\left[X_{n}, X_{n+1}-X_{n+1}^{*}\right]^{*}\right) .
\end{aligned}
$$

Using this the proof is just a simple adaptation of the proof of Theorem 2.3 . Here one also has to note that every element in $\mathcal{K}$ is of the form $a-a^{*}, a \in \mathcal{A}$.

\section{Lie Ideals and Lie Skew-Ideals}

The aim of this section is to describe Lie ideals and Lie skew-ideals in finite dimensional central simple algebras, and then extend these descriptions (in an appropriate way) to prime PI algebras. Let us recall that an algebra is said to be prime if the product of any two of its nonzero ideals is nonzero. Prime PI algebras can be embedded (in a particularly nice way) into finite dimensional central simple algebras, so these extensions are rather straightforward. Nevertheless, we have decided to consider also prime PI algebras because there exist important examples of such algebras that are not simple - for instance, the algebra of generic matrices considered in Section 5 .

The concept of a Lie ideal is a classical one, and the result obtained in the first subsection below is not particularly surprising. The bulk of the section is devoted to Lie skew-ideals.

3.1. Lie ideals in finite dimensional central simple algebras. The following result is folklore.

Lemma 3.1. Let $\mathcal{A}=M_{d}(\mathbb{F}), d \geq 2$, and suppose that $d \neq 2$ or $\operatorname{char}(\mathbb{F}) \neq 2$. Then $\mathcal{A}$ has exactly four Lie ideals: $0, \mathcal{Z},[\mathcal{A}, \mathcal{A}]$ and $\mathcal{A}$.

Here, the center $\mathcal{Z}$ is equal to $\mathbb{F}$, the set of all scalar matrices, and $[\mathcal{A}, \mathcal{A}]$ is the set of all commutators $[A, B], A, B \in \mathcal{A}$, or equivalently, the set of all matrices with zero trace.

A general remark about notation: if $\mathcal{U}$ and $\mathcal{V}$ are subspaces of an algebra $\mathcal{A}$, then by $[\mathcal{U}, \mathcal{V}]$ we denote the linear span of all commutators $[u, v], u \in \mathcal{U}, v \in \mathcal{V}$. By chance in the case of $\mathcal{A}=M_{d}(\mathbb{F})$ the linear space $[\mathcal{A}, \mathcal{A}]$ coincides with the set of all commutators $[A, B][\mathrm{Sho}, \widehat{\mathrm{AM}}]$, but in general this is not true.

One can prove Lemma 3.1 by a direct computation. On the other hand, the lemma follows immediately from a substantially more general result by Herstein Her2, 
Theorem 1.5] stating that under very mild assumptions a Lie ideal of a simple algebra $\mathcal{A}$ either contains $[\mathcal{A}, \mathcal{A}]$ or is contained in $\mathcal{Z}$. We also remark that the case when $d=2$ and $\operatorname{char}(\mathbb{F})=2$ is really exceptional, see [Her2, p. 6].

Lemma 3.1 can be quite easily extended to more general algebras. We begin with

Proposition 3.2. Let $\mathcal{A}$ be a finite dimensional central simple algebra such that $\operatorname{dim}_{\mathbb{F}} \mathcal{A} \neq 4$ or $\operatorname{char}(\mathbb{F}) \neq 2$. If $\mathcal{L}$ is a Lie ideal of $\mathcal{A}$, then $\mathcal{L}$ is either $0, \mathcal{Z},[\mathcal{A}, \mathcal{A}]$ or $\mathcal{A}$.

Proof. Let $\overline{\mathcal{Z}}$ be the algebraic closure of $\mathcal{Z}=\mathbb{F}$. We now form the scalar extension $\overline{\mathcal{A}}=$ $\mathcal{A} \otimes_{\mathcal{Z}} \overline{\mathcal{Z}}$ which is, as a finite dimensional central simple algebra over an algebraically closed field $\overline{\mathcal{Z}}$, isomorphic to $M_{d}(\overline{\mathcal{Z}})$ where $d=\sqrt{\operatorname{dim}_{\mathcal{Z}} \mathcal{A}}$. Thus $d \neq 2$ if $\operatorname{dim}_{\mathcal{Z}} \mathcal{A} \neq 4$.

Observe that $\overline{\mathcal{L}}=\mathcal{L} \otimes \overline{\mathcal{Z}}$ is a Lie ideal of $\overline{\mathcal{A}}$. Lemma 3.1 tells us that $\overline{\mathcal{L}}$ is either 0 , $\overline{\mathcal{Z}},[\overline{\mathcal{A}}, \overline{\mathcal{A}}]$ or $\overline{\mathcal{A}}$. Note that

$$
0=0 \otimes \overline{\mathcal{Z}}, \quad \overline{\mathcal{Z}}=\mathcal{Z} \otimes \overline{\mathcal{Z}}, \quad[\overline{\mathcal{A}}, \overline{\mathcal{A}}]=[\mathcal{A}, \mathcal{A}] \otimes \overline{\mathcal{Z}}, \quad \overline{\mathcal{A}}=\mathcal{A} \otimes \overline{\mathcal{Z}} .
$$

We now make a small digression and record the following easily proven fact: if $\mathcal{W}$ and $\mathcal{V}$ are subspaces of $\mathcal{A}$ and $\mathcal{W} \otimes \overline{\mathcal{Z}}=\mathcal{V} \otimes \overline{\mathcal{Z}}$, then $\mathcal{W}=\mathcal{V}$. Accordingly, since $\overline{\mathcal{L}}=\mathcal{L} \otimes \overline{\mathcal{Z}}$ is equal to one of the sets listed in (4), it follows that $\mathcal{L}$ is either $0, \mathcal{Z}$, $[\mathcal{A}, \mathcal{A}]$ or $\mathcal{A}$.

Now assume that $\mathcal{A}$ is a prime PI algebra. Then $\mathcal{Z} \neq 0$ and the central closure (i.e., a central localization, also called the algebra of central quotients [Row, §1.7]) $\widetilde{\mathcal{A}}$ of $\mathcal{A}$ consists of elements of the form $z^{-1} a$ where $a \in \mathcal{A}$ and $0 \neq z \in \mathcal{Z}$. Furthermore, $\widetilde{\mathcal{A}}$ is a finite dimensional central simple algebra over the field of fractions $\widetilde{\mathcal{Z}}$ of $\mathcal{Z}$. This is a version of Posner's theorem together with Rowen's sharpening, see for example [Row, Theorem 1.7.9]. Of course, $\widetilde{\mathcal{Z}}$ is a field extension of $\mathbb{F}$ and so they have the same characteristic. Given a subset $\mathcal{V}$ of $\mathcal{A}$, we shall write $\widetilde{\mathcal{V}}$ for the linear span of $\mathcal{V}$ over $\widetilde{\mathcal{Z}}$.

Corollary 3.3. Let $\mathcal{A}$ be a prime PI algebra such that $\operatorname{dim}_{\widetilde{\mathcal{Z}}} \widetilde{\mathcal{A}} \neq 4$ or $\operatorname{char}(\mathbb{F}) \neq 2$. If $\mathcal{L}$ is a Lie ideal of $\mathcal{A}$, then $\widetilde{\mathcal{L}}$ is either $0, \widetilde{\mathcal{Z}},[\widetilde{\mathcal{A}}, \widetilde{\mathcal{A}}]$ or $\widetilde{\mathcal{A}}$.

Proof. Noticing that $\widetilde{\mathcal{L}}$ is a Lie ideal of $\widetilde{\mathcal{A}}$, the result follows immediately from Proposition 3.2 .

For more details about Lie ideals in simple algebras we refer the reader to Her2]. A more recent reference is the paper [BKS] in which Lie ideals are thoroughly studied in both algebraic and analytic setting.

3.2. General remarks on Lie skew-ideals. Let $\mathcal{A}$ be a $*$-algebra over a field $\mathbb{F}$ with $\operatorname{char}(\mathbb{F}) \neq 2$. Every Lie ideal of $\mathcal{A}$ is also a Lie skew-ideal of $A$, while the converse is not true in general. For example, $\mathcal{S}$ and $\mathcal{K}$ are Lie skew-ideals, which are only rarely Lie ideals. Obviously, Lie skew-ideals are closed under sums and intersections. Further, if $\mathcal{L}_{1}$ and $\mathcal{L}_{2}$ are Lie skew-ideals, then $\left[\mathcal{L}_{1}, \mathcal{L}_{2}\right]$ is also a Lie skew-ideal. This can be easily checked by using the Jacobi identity.

Let us mention eight examples of Lie skew-ideals: $0, \mathcal{Z}, \mathcal{K},[\mathcal{S}, \mathcal{K}], \mathcal{S}, \mathcal{Z}+\mathcal{K}$, $[\mathcal{A}, \mathcal{A}]$, and $\mathcal{A}$. As indicated above, there are other natural examples. The reasons for pointing out these eight examples will become clear in the sequel. 
For subspaces of $\mathcal{K}$ the notion of a Lie skew-ideal coincides with the standard and extensively studied notion of a Lie ideal of $\mathcal{K}$. For a simple algebra $\mathcal{A}$ with $\operatorname{dim}_{\mathcal{Z}} \mathcal{A}>16$, a classical theorem by Herstein states that every Lie ideal of $\mathcal{K}$ either contains $[\mathcal{K}, \mathcal{K}]$ or is contained in $\mathcal{Z}[$ Her2, Theorem 2.12]. The following example justifies the dimension restriction.

Example 3.4. If $\mathcal{A}=M_{4}(\mathbb{F}), \operatorname{char}(\mathbb{F}) \neq 2$, endowed with the transpose involution, then $\mathcal{K}$ can can be written as a Lie theoretic direct sum of two simple Lie algebras, $\mathcal{K}=\mathcal{K}_{1} \oplus \mathcal{K}_{2}$. Each $\mathcal{K}_{i}$ is 3-dimensional; a basis of $\mathcal{K}_{1}$ is $\left\{E_{12}-E_{21}+E_{34}-E_{43}, E_{13}-\right.$ $\left.E_{31}+E_{42}-E_{24}, E_{14}-E_{41}+E_{23}-E_{32}\right\}$, and a basis of $\mathcal{K}_{2}$ is $\left\{E_{12}-E_{21}-E_{34}+\right.$ $\left.E_{43}, E_{13}-E_{31}-E_{42}+E_{24}, E_{14}-E_{41}-E_{23}+E_{32}\right\}$. Thus, $\mathcal{K}_{1}$ and $\mathcal{K}_{2}$ are Lie ideals of $\mathcal{K}$ (and hence Lie skew-ideals of $\mathcal{A}$ ) which are neither contained in $\mathcal{Z}$ nor do they contain $[\mathcal{K}, \mathcal{K}]$ (which is equal to $\mathcal{K}$ in this example).

Somewhat less known is Herstein's result which treats linear subspaces $\mathcal{L}$ of $\mathcal{S}$ satisfying $[\mathcal{L},[\mathcal{K}, \mathcal{K}]] \subseteq \mathcal{L}$ Her1, Theorem 2.1]. Again assuming the simplicity of $\mathcal{A}$ and some additional mild technical conditions, this result says that $\mathcal{L}$ either contains $[\mathcal{S}, \mathcal{K}]$ or is contained in $\mathcal{Z}$. Of course this result also covers Lie skew-ideals of $\mathcal{A}$ that are contained in $\mathcal{S}$.

Now let $\mathcal{L}$ be a general Lie skew-ideal. If $\mathcal{L}^{*}=\mathcal{L}$ then $\mathcal{L}=\mathcal{L} \cap \mathcal{S} \oplus \mathcal{L} \cap \mathcal{K}$, and for $\mathcal{L} \cap \mathcal{S}$ and $\mathcal{L} \cap \mathcal{K}$ we can use Herstein's aforementioned results. However, not every Lie skew-ideal is invariant under $*$.

Example 3.5. Let $\mathcal{L}$ be the one-dimensional subspace of $M_{2}(\mathbb{F})$ generated by $L=$ $E_{11}+E_{12}-E_{21}+E_{22}$. Note that $\mathcal{L}$ is a Lie skew-ideal of $M_{2}(\mathbb{F})$ with respect to the transpose involution, but is not invariant under this involution.

In what follows we shall see that this example is a rather exceptional one. Nevertheless, it seems that Herstein's theorems are not directly applicable to our purposes. Not only because of the *-invariance problem, but also since we wish to obtain a precise description of all Lie skew-ideals rather than just information about certain inclusions. This seems to be out of reach in such a general class as is the class of simple algebras. But we shall confine ourselves to the more special class of finite dimensional simple algebras. Still, Herstein's theory has been useful for us philosophically. It indicates that Lie skew-ideals are treatable.

Let us briefly discuss another question that naturally appears in connection with Lie skew-ideals, and which we find interesting in its own right. Assume that $\mathcal{A}$ has an identity element 1 (in general we do not assume this in advance) and let $\mathcal{U}$ be the set of all unitary elements in $\mathcal{A}, \mathcal{U}=\left\{u \in \mathcal{A} \mid u^{*}=u^{-1}\right\}$. This question concerns the relation between Lie skew-ideals and subspaces of $\mathcal{A}$ that are closed under conjugation with unitary elements, i.e., subspaces $\mathcal{L}$ of $\mathcal{A}$ such that $u \mathcal{L} u^{*} \subseteq \mathcal{L}$ for every $u \in \mathcal{U}$. This is an analogue to the problem of the relation between Lie ideals and subspaces closed under conjugation with invertible elements (in other words, subspaces invariant under all inner automorphisms). One of the basic results in the latter area says that a closed linear subspace of a Banach algebra must be a Lie ideal if it closed under conjugation with invertible elements. The same proof shows the following.

Proposition 3.6. Let $\mathcal{A}$ be a real or complex Banach algebra with $\mathbb{R}$-linear involution *. If a closed linear subspace $\mathcal{L}$ of $\mathcal{A}$ is closed under conjugation with unitaries, then $\mathcal{L}$ is a Lie skew-ideal of $\mathcal{A}$. 
Proof. If $k \in \mathcal{K}$, then $e^{t k} \in \mathcal{U}$ for every $t \in \mathbb{R}$. Therefore, for every $t \neq 0$ and $x \in \mathcal{L}$, $\mathcal{L}$ contains the element

$$
\frac{1}{t}\left(e^{t k} x\left(e^{t k}\right)^{*}-x\right)=\frac{1}{t}\left(e^{t k} x e^{-t k}-x\right)=[k, x]+\frac{t}{2 !}[k,[k, x]]+\frac{t^{2}}{3 !}[k,[k,[k, x]]]+\ldots
$$

(The second equality is a special case of the Baker-Campbell-Hausdorff formula.) Since $\mathcal{L}$ is closed it follows that

$$
[k, x]=\lim _{t \rightarrow 0} \frac{1}{t}\left(e^{t k} x\left(e^{t k}\right)^{*}-x\right) \in \mathcal{L} .
$$

Algebraic versions of this propositions cannot be obtained so easily. Namely, in a purely algebraic setting the set of unitary elements can be very small, and so $u \mathcal{L} u^{*} \subseteq \mathcal{L}$ for all $u \in \mathcal{U}$ may trivially hold.

\section{Example 3.7.}

(1) If $\mathcal{A}=\mathbb{F}\left\langle\bar{X}, \bar{X}^{*}\right\rangle$ is a free $*$-algebra, then $\mathcal{U} \subseteq \mathbb{F}$, and so every subspace of $\mathcal{A}$ is closed under conjugation with unitaries. But of course not every subspace is a Lie skew-ideal.

(2) For a finite dimensional example of characteristic 3, let $\mathbb{F}_{3}=\{0,1,2\}$ denote the field on 3 elements and consider $M_{2}\left(\mathbb{F}_{3}\right)$ endowed with the transpose involution. Then $\mathcal{K}$ is spanned by $k=\left[\begin{array}{ll}0 & 2 \\ 1 & 0\end{array}\right]$ and

$$
\mathcal{U}=\left\{\left[\begin{array}{cc}
\lambda & 0 \\
0 & \mu
\end{array}\right],\left[\begin{array}{cc}
0 & \lambda \\
\mu & 0
\end{array}\right] \mid \lambda, \mu \in \mathbb{F}_{3} \backslash\{0\}\right\} .
$$

Now it is easy to construct examples of subspaces closed under unitary conjugation that are not Lie skew-ideals. For instance, take $\mathbb{F}_{3}\left[\begin{array}{ll}0 & 1 \\ 1 & 0\end{array}\right]$.

(3) We conclude by presenting an example of a slightly different flavor. Cohn Coh Exercise 2.1.10] has constructed a division algebra (necessarily of characteristic 2 ) with only one unitary element. Like in (1) this gives rise to an abundance of examples of subspaces closed under unitary conjugation that are not Lie skewideals.

There is, however, the following nice result by Lanski (which we state using our terminology): If $\mathcal{A}$ is an algebraic $*$-algebra over an infinite field $\mathbb{F}$ with $\operatorname{char}(\mathbb{F}) \neq$ 2 , then every linear subspace of $\mathcal{A}$ which is closed under conjugation with unitary elements is a Lie skew-ideal of $\mathcal{A}[\mathrm{Lan}$, Theorem 1]. The converse is not true. Indeed, one can check that Lie skew-ideals from Examples 3.4 and 3.5 are not closed under conjugation with unitaries. On the other hand, Lie skew-ideals that will be important for us, namely $0, \mathcal{Z}, \mathcal{K},[\mathcal{S}, \mathcal{K}], \mathcal{S}, \mathcal{Z}+\mathcal{K},[\mathcal{A}, \mathcal{A}]$ and $\mathcal{A}$, are all closed under conjugation with unitaries.

Let us finally mention that for every $f \in \mathbb{F}\langle\bar{X}\rangle$ and every algebra $\mathcal{A}$, span $f(\mathcal{A})$ is closed under conjugation with invertible elements; moreover, it is invariant under every algebra endomorphism of $\mathcal{A}$. Similarly, for every $f \in \mathbb{F}\left\langle\bar{X}, \bar{X}^{*}\right\rangle$, $\operatorname{span} f(\mathcal{A})$ is closed under conjugation with unitary elements, and moreover, it is invariant under every algebra $*$-endomorphism of $\mathcal{A}$. In view of these observations we have been in fact hesitating at the early stage of this work whether the definition of a Lie skew-ideal 
should also involve the conjugation with unitaries. However, it has turned out that this would lead to certain technical difficulties, and so we have decided to focus on commutation with skew-symmetric elements only.

3.3. Lie skew-ideals in matrix algebras. The purpose of this section is to describe Lie skew-ideals in matrix algebras with respect to two basic involutions, the transpose and the usual symplectic involution. Let us at the beginning present these notions in a more general framework.

Definition 3.8. Let $\mathcal{A}$ be a central simple $*$-algebra of degree $d$, i.e., of dimension $d^{2}$ over its center $\mathcal{Z}$. Then $*$ is called orthogonal if

$$
\operatorname{dim}_{\mathcal{Z}} \mathcal{S}=\frac{d(d+1)}{2}
$$

and symplectic if

$$
\operatorname{dim}_{\mathcal{Z}} \mathcal{S}=\frac{d(d-1)}{2} .
$$

Symplectic involutions only exist for even $d$. For a full account on (central simple) algebras with involutions we refer the reader to [KMRT.

The basic example of an orthogonal involution on the algebra $\mathcal{A}=M_{d}(\mathbb{F})$ is the transpose involution, $A \mapsto A^{t}$. The usual symplectic involution on $\mathcal{A}=M_{d}(\mathbb{F})$ is defined when $d$ is even, $d=2 d_{0}$, as follows:

$$
\left[\begin{array}{ll}
A & B \\
C & D
\end{array}\right]^{*}=\left[\begin{array}{cc}
D^{t} & -B^{t} \\
-C^{t} & A^{t}
\end{array}\right] \quad \text { where } A, B, C, D \in M_{d_{0}}(\mathbb{F}) .
$$

Definition 3.9. An involution on an algebra $\mathcal{A}$ is said to be of the first kind if it fixes $\mathcal{Z}$ pointwise and of the second kind otherwise. Involutions of the second kind are also called unitary involutions.

Both the transpose and the usual symplectic involution are of course involutions of the first kind.

Lemma 3.10. Let $\mathcal{A}=M_{d}(\mathbb{F})$ be endowed with the transpose involution, and let $\operatorname{char}(\mathbb{F}) \neq 2,3$. If $d \neq 2,4$, then $0, \mathcal{Z}, \mathcal{K},[\mathcal{S}, \mathcal{K}], \mathcal{S}, \mathcal{Z}+\mathcal{K},[\mathcal{A}, \mathcal{A}]$, and $\mathcal{A}$ are the only Lie skew-ideals of $\mathcal{A}$.

Proof. Let us begin by noting that $\mathcal{Z}$ consists of all scalar matrices, $[\mathcal{S}, \mathcal{K}]$ consists of all symmetric matrices with trace 0 , and $[\mathcal{A}, \mathcal{A}]$ consists of all matrices with trace 0 .

Since $d \neq 2,4, \mathcal{K}$ is a simple Lie algebra. This is well-known and easy to see (see for example BMM, p. 443]). Given a Lie skew-ideal $\mathcal{L}$ of $\mathcal{A}$, we have that $\mathcal{L} \cap \mathcal{K}$ is a Lie ideal of $\mathcal{K}$, and hence either $\mathcal{L} \cap \mathcal{K}=0$ or $\mathcal{L} \cap \mathcal{K}=\mathcal{K}$. That is,

$$
\mathcal{L} \cap \mathcal{K}=0 \quad \text { or } \quad \mathcal{K} \subseteq \mathcal{L} .
$$

Let us first consider the case where $\mathcal{L} \subseteq \mathcal{Z}+\mathcal{K}$. If $\mathcal{L} \subseteq \mathcal{Z}$, then of course either $\mathcal{L}=0$ or $\mathcal{L}=\mathcal{Z}$. If $\mathcal{L} \nsubseteq \mathcal{Z}$, then $\mathcal{L}$ contains a matrix $\lambda I+K_{0}$ where $\lambda \in \mathbb{F}$ and $0 \neq K_{0} \in \mathcal{K}$. Picking $K_{1} \in \mathcal{K}$ which does not commute with $K_{0}$ it follows that $0 \neq\left[K_{0}, K_{1}\right]=\left[\lambda I+K_{0}, K_{1}\right] \in \mathcal{L} \cap \mathcal{K}$. Therefore $\mathcal{K} \subseteq \mathcal{L}$ by (5). But then either $\mathcal{L}=\mathcal{K}$ or $\mathcal{L}=\mathcal{Z}+\mathcal{K}$. 
Assume from now on that $\mathcal{L} \nsubseteq \mathcal{Z}+\mathcal{K}$. Therefore there exists $A=\left(a_{i j}\right) \in \mathcal{L}$ such that for some $i \neq j$, either $\alpha=a_{j j}-a_{i i} \neq 0$ or $\beta=a_{i j}+a_{j i} \neq 0$. Since for every $K \in \mathcal{K}$ also $K^{3} \in \mathcal{K}$, we have

$$
K^{2} A K-K A K^{2}=\frac{1}{3}\left([[[A, K], K], K]-\left[A, K^{3}\right]\right) \in \mathcal{L} .
$$

For $K=E_{i j}-E_{j i}$ we get

$$
\alpha\left(E_{i j}+E_{j i}\right)+\beta\left(E_{i i}-E_{j j}\right) \in \mathcal{L} .
$$

Pick $k$ different from $i$ and $j$ (recall that $d \neq 2$ !). Since $E_{j k}-E_{k j} \in \mathcal{L}$, it follows that $\mathcal{L}$ contains

$\left[\left[\alpha\left(E_{i j}+E_{j i}\right)+\beta\left(E_{i i}-E_{j j}\right), E_{j k}-E_{k j}\right], E_{j k}-E_{k j}\right]=-\alpha\left(E_{i j}+E_{j i}\right)+2 \beta\left(E_{j j}-E_{k k}\right)$.

Using this together with (6) it follows that $\beta\left(E_{i i}+E_{j j}-2 E_{k k}\right) \in \mathcal{L}$, and hence also

$$
\beta\left(E_{i k}+E_{k i}\right)=\frac{1}{3}\left[\beta\left(E_{i i}+E_{j j}-2 E_{k k}\right), E_{i k}-E_{k i}\right] \in \mathcal{L} .
$$

If $\beta \neq 0$, then this yields. $E_{i k}+E_{k i} \in \mathcal{L}$. If, however, $\beta=0$, then $\alpha \neq 0$ and hence $E_{i j}+E_{j i} \in \mathcal{L}$ by (6). Thus, in any case $\mathcal{L}$ contains a matrix of the form $E_{u v}+E_{v u}$ with $u \neq v$. We claim that this implies that $\mathcal{L}$ contains all matrices of the form $E_{p q}+E_{q p}$ with $p \neq q$. Indeed, if $\{p, q\} \cap\{u, v\}=\emptyset$, then this follows from $E_{p q}+E_{q p}=\left[\left[E_{u v}+E_{v u}, E_{v p}-E_{p v}\right], E_{u q}-E_{q u}\right]$, and if $\{p, q\} \cap\{u, v\} \neq \emptyset$, then the proof is even easier. Consequently, $E_{q q}-E_{p p}=\frac{1}{2}\left[E_{p q}+E_{q p}, E_{p q}-E_{q p}\right] \in \mathcal{L}$. Note that all these relations can be summarized as

$$
[\mathcal{S}, \mathcal{K}] \subseteq \mathcal{L}
$$

Suppose that $\mathcal{L} \cap \mathcal{K}=0$. We claim that in this case $\mathcal{L} \subseteq \mathcal{S}$. Indeed, if this was not true, then $\mathcal{L}$ would contain a matrix $K_{0}+S_{0}$ with $0 \neq K_{0} \in \mathcal{K}$ and $S_{0} \in \mathcal{S}$. Picking $K_{1} \in \mathcal{K}$ that does not commute with $K_{0}$ it then follows from 77 that $0 \neq\left[K_{0}, K_{1}\right]=$ $\left[K_{0}+S_{0}, K_{1}\right]-\left[S_{0}, K_{1}\right] \in \mathcal{L} \cap \mathcal{K}$, a contradiction. Thus $[\mathcal{S}, \mathcal{K}] \subseteq \mathcal{L} \subseteq \mathcal{S}$ and so either $\mathcal{L}=[\mathcal{S}, \mathcal{K}]$ or $\mathcal{L}=\mathcal{S}$.

It remains to consider the case where $\mathcal{L} \cap \mathcal{K} \neq 0$. In this case $\mathcal{K} \subseteq \mathcal{L}$ by (5). Since $\mathcal{L}$ also contains $[\mathcal{S}, \mathcal{K}]$ and since $[\mathcal{S}, \mathcal{K}]+\mathcal{K}=[\mathcal{A}, \mathcal{A}]$, it follows that $[\mathcal{A}, \mathcal{A}] \subseteq \mathcal{L} \subseteq \mathcal{A}$. But then either $\mathcal{L}=[\mathcal{A}, \mathcal{A}]$ or $\mathcal{L}=\mathcal{A}$.

The cases where $d=2$ or $d=4$ are indeed exceptional; see Examples 3.5 and 3.4

Our next aim is to prove a version of Lemma 3.10 for the usual symplectic involution. For this we need the following lemma which describes the structure of certain subspaces of $M_{d}(\mathbb{F})$ that are in particular Lie skew-ideals of $M_{d}(\mathbb{F})$ with respect to the transpose involution. Since the restrictions $d \neq 2,4$ and $\operatorname{char}(\mathbb{F}) \neq 3$ are unnecessary in this situation, we cannot apply Lemma 3.10 . In any case a direct computational proof could be easily given. However, a result by Montgomery Mon Corollary 1] describing additive subgroups $\mathcal{M}$ of simple rings $\mathcal{A}$ with involution satisfying $a \mathcal{M} a^{*} \subseteq \mathcal{M}$ for all $a \in \mathcal{A}$ will make it possible for us to use a shortcut. This result implies that if $\mathcal{A}$ is a simple algebra over a field $\mathbb{F}$ with $\operatorname{char}(\mathbb{F}) \neq 2$, the involution $*$ is of the first kind, and $\mathcal{M}$ is such a linear subspace of $\mathcal{A}$, then $\mathcal{M}$ must be either $0, \mathcal{K}, \mathcal{S}$, or $\mathcal{A}$. 
Lemma 3.11. Let $\mathcal{A}=M_{d}(\mathbb{F})$ be endowed with the transpose involution, and let $\operatorname{char}(\mathbb{F}) \neq 2$. If $\mathcal{M}$ is a linear subspace of $\mathcal{A}$ such that $M A^{t}+A M \in \mathcal{M}$ for all $M \in \mathcal{M}$ and $A \in \mathcal{A}$, then $\mathcal{M}$ is either $0, \mathcal{K}, \mathcal{S}$, or $\mathcal{A}$.

Proof. From the identity

$$
A M A^{t}=\frac{1}{2}\left(\left(\left(M A^{t}+A M\right) A^{t}+A\left(M A^{t}+A M\right)\right)-\left(M\left(A^{2}\right)^{t}+A^{2} M\right)\right)
$$

it follows that $A M A^{t} \in \mathcal{M}$ for all $A \in \mathcal{A}$ and $M \in \mathcal{M}$. Therefore the result follows immediately from [Mon, Corollary 1].

Lemma 3.12. Let $\mathcal{A}=M_{2 d_{0}}(\mathbb{F})$, let $*$ be the usual symplectic involution on $\mathcal{A}$, and let $\operatorname{char}(\mathbb{F}) \neq 2$. Then $0, \mathcal{Z}, \mathcal{K},[\mathcal{S}, \mathcal{K}], \mathcal{S}, \mathcal{Z}+\mathcal{K},[\mathcal{A}, \mathcal{A}]$, and $\mathcal{A}$ are the only Lie skew-ideals of $\mathcal{A}$.

Proof. Set $\mathcal{A}_{0}=M_{d_{0}}(\mathbb{F})$ and let $\mathcal{K}_{0}$ and $\mathcal{S}_{0}$ denote the sets of symmetric and skewsymmetric matrices in $\mathcal{A}_{0}$ with respect to the transpose involution. Note that $\mathcal{K}$ consists of all matrices of the form

$$
\left[\begin{array}{cc}
A & S \\
T & -A^{t}
\end{array}\right] \quad \text { where } A \in \mathcal{A}_{0}, S, T \in \mathcal{S}_{0},
$$

and $\mathcal{S}$ consists of all matrices of the form

$$
\left[\begin{array}{cc}
A & K \\
L & A^{t}
\end{array}\right] \quad \text { where } A \in \mathcal{A}_{0}, K, L \in \mathcal{K}_{0}
$$

Let $\mathcal{L}$ be a Lie skew-ideal of $\mathcal{A}$, and let $\left[\begin{array}{ll}A & B \\ C & D\end{array}\right] \in \mathcal{L}$. Commuting this matrix with $\left[\begin{array}{cc}I & 0 \\ 0 & -I\end{array}\right] \in \mathcal{K}$ it follows that $\left[\begin{array}{cc}0 & -B \\ C & 0\end{array}\right] \in \mathcal{L}$. Furthermore, commuting the latter matrix with $\left[\begin{array}{cc}I & 0 \\ 0 & -I\end{array}\right]$ one easily shows that actually both $\left[\begin{array}{cc}0 & B \\ 0 & 0\end{array}\right]$ and $\left[\begin{array}{ll}0 & 0 \\ C & 0\end{array}\right]$ belong to $\mathcal{L}$. Thus, we have

$$
\left[\begin{array}{ll}
A & B \\
C & D
\end{array}\right] \in \mathcal{L} \Rightarrow\left[\begin{array}{cc}
A & 0 \\
0 & D
\end{array}\right],\left[\begin{array}{cc}
0 & B \\
0 & 0
\end{array}\right],\left[\begin{array}{ll}
0 & 0 \\
C & 0
\end{array}\right] \in \mathcal{L}
$$

Let $\mathcal{M}_{0}$ be the set of all $M \in \mathcal{A}_{0}$ such that $\left[\begin{array}{cc}0 & M \\ 0 & 0\end{array}\right] \in \mathcal{L}$. Commuting this matrix with $\left[\begin{array}{cc}A & 0 \\ 0 & -A^{t}\end{array}\right] \in \mathcal{K}$ it follows that $\mathcal{M}_{0}$, considered as a subspace of $\mathcal{A}_{0}$, satisfies the condition of Lemma 3.11. Therefore $\mathcal{M}_{0}$ is $0, \mathcal{K}_{0}, \mathcal{S}_{0}$, or $\mathcal{A}_{0}$. Each of these four cases shall be considered separately.

Assume that $\mathcal{M}_{0}=0$. From (8) we see that then any matrix in $\mathcal{L}$ is of the form $\left[\begin{array}{lr}A & 0 \\ C & D\end{array}\right]$. Commuting such a matrix with $\left[\begin{array}{ll}0 & S \\ 0 & 0\end{array}\right] \in \mathcal{K}$ it follows that $A S=S D$ for all $S \in \mathcal{S}_{0}$. It is easy to see that this is possible only if $A=D$ is a scalar matrix. Consequently, commuting $\left[\begin{array}{ll}0 & 0 \\ C & 0\end{array}\right]$ with $\left[\begin{array}{ll}0 & I \\ 0 & 0\end{array}\right]$ it follows that $C=-C$, i.e., $C=0$. Therefore $\mathcal{L}$ consists only of scalar matrices. There are just two possibilities: either $\mathcal{L}=0$ or $\mathcal{L}=\mathcal{Z}$. 
Next we consider the case where $\mathcal{M}_{0}=\mathcal{K}_{0}$. Pick $K \in \mathcal{K}_{0}$ and $S \in \mathcal{S}_{0}$. Commuting $\left[\begin{array}{cc}0 & K \\ 0 & 0\end{array}\right] \in \mathcal{L}$ with $\left[\begin{array}{ll}0 & 0 \\ S & 0\end{array}\right] \in \mathcal{K}$ it follows that $\left[\begin{array}{cc}K S & 0 \\ 0 & -S K\end{array}\right] \in \mathcal{L}$. It is easy to see that every matrix in $\mathcal{A}_{0}$ of the form $K S$ has trace 0 , and conversely, every matrix in $\mathcal{A}_{0}$ with trace 0 is a linear span of matrices of the form $K S$. Therefore $\mathcal{L}$ contains all matrices $\left[\begin{array}{cc}A & 0 \\ 0 & A^{t}\end{array}\right]$ with $A \in\left[\mathcal{A}_{0}, A_{0}\right]$. Now take any matrix in $\mathcal{L}$ of the form $\left[\begin{array}{cc}A & 0 \\ 0 & D\end{array}\right]$. Its commutator with $\left[\begin{array}{ll}0 & 0 \\ S & 0\end{array}\right] \in \mathcal{K}$ is $\left[\begin{array}{cc}0 & A S-S D \\ 0 & 0\end{array}\right]$. Since this matrix must be in $\mathcal{L}$ it follows that $A S-S D \in \mathcal{K}_{0}$ for every $S \in \mathcal{S}_{0}$. This condition can be rewritten as $S\left(A^{t}-D\right)+\left(A^{t}-D\right)^{t} S=0$ for every $S \in \mathcal{S}_{0}$. It is easy to see that this forces $A^{t}=D$. Therefore the "diagonal part" of $\mathcal{L}$ consists only of matrices of the form $\left[\begin{array}{cc}A & 0 \\ 0 & A^{t}\end{array}\right]$, and there are two possibilities: either all such matrices with an arbitrary $A \in \mathcal{A}_{0}$ are in $\mathcal{L}$, or only all such matrices with the restriction that $A$ has trace 0 , i.e., $A \in\left[\mathcal{A}_{0}, \mathcal{A}_{0}\right]$. It remains to examine the "lower corner" part. Pick $\left[\begin{array}{ll}0 & 0 \\ C & 0\end{array}\right] \in \mathcal{L}$. Commuting it with $\left[\begin{array}{ll}0 & I \\ 0 & 0\end{array}\right] \in \mathcal{K}$ we get $\left[\begin{array}{cc}-C & 0 \\ 0 & C\end{array}\right] \in \mathcal{L}$. But then $C$ must lie in $\mathcal{K}_{0}$. Conversely, as the commutator of $\left[\begin{array}{cc}A & 0 \\ 0 & A^{t}\end{array}\right] \in \mathcal{L}$ with $\left[\begin{array}{ll}0 & 0 \\ I & 0\end{array}\right] \in \mathcal{K}$ is $\left[\begin{array}{cc}0 & 0 \\ A^{t}-A & 0\end{array}\right]$, and since every $K \in \mathcal{K}_{0}$ can be written as $K=A^{t}-A$ with $A \in\left[\mathcal{A}_{0}, \mathcal{A}_{0}\right]$, it follows that $\mathcal{L}$ contains all matrices $\left[\begin{array}{cc}0 & 0 \\ K & 0\end{array}\right]$ with $K \in \mathcal{K}_{0}$. We can now gather all the information derived in the following conclusion: $\mathcal{L}$ either consists of all matrices $\left[\begin{array}{cc}A & K \\ L & A^{t}\end{array}\right]$ with $A \in \mathcal{A}_{0}, S, T \in \mathcal{K}_{0}$ or of all such matrices with $A \in\left[\mathcal{A}_{0}, A_{0}\right], S, T \in \mathcal{K}_{0}$. In the first case $\mathcal{L}=\mathcal{S}$ and in the second case $\mathcal{L}=[\mathcal{S}, \mathcal{K}]$.

The cases where $\mathcal{M}_{0}=\mathcal{S}_{0}$ or $\mathcal{M}_{0}=\mathcal{A}_{0}$ can be treated similarly as the $\mathcal{M}_{0}=\mathcal{K}_{0}$ case. One can show that $\mathcal{M}_{0}=\mathcal{S}_{0}$ implies that $\mathcal{L}=\mathcal{K}$ or $\mathcal{L}=\mathcal{Z}+\mathcal{K}$, and $\mathcal{M}_{0}=\mathcal{A}_{0}$ implies that $\mathcal{L}=[\mathcal{A}, \mathcal{A}]$ or $\mathcal{L}=\mathcal{A}$. There are some differences compared to the case just treated, but the necessary modifications are quite obvious. Therefore we omit the details.

3.4. Lie skew-ideals in finite dimensional central simple algebras. The above results make it possible for us to describe Lie skew-ideals in finite dimensional central simple algebras with involution. The description depends on the kind of an involution.

Theorem 3.13. Let $\mathcal{A}$ be a finite dimensional central simple algebra with involution of the first kind, and let $\mathcal{L}$ be a Lie skew-ideal of $\mathcal{A}$. If $\operatorname{dim}_{\mathbb{F}} \mathcal{A} \neq 4,16$ and $\operatorname{char}(\mathbb{F}) \neq 2,3$, then $\mathcal{L}$ is either $0, \mathcal{Z}, \mathcal{K},[\mathcal{S}, \mathcal{K}], \mathcal{S}, \mathcal{Z}+\mathcal{K},[\mathcal{A}, \mathcal{A}]$ or $\mathcal{A}$.

Proof. As in the proof of Proposition 3.2 we denote the algebraic closure of $\mathcal{Z}=\mathbb{F}$ by $\overline{\mathcal{Z}}$, and form the scalar extension $\overline{\mathcal{A}}=\mathcal{A} \otimes_{\mathcal{Z}} \overline{\mathcal{Z}}$ which is isomorphic to $M_{d}(\overline{\mathcal{Z}})$ where $d=\sqrt{\operatorname{dim}_{\mathbb{F}} \mathcal{A}}$. We can extend $*$ to an involution (also of the first kind) of $\overline{\mathcal{A}}$ by $(a \otimes \lambda)^{*}=a^{*} \otimes \lambda$. Note that the set of symmetric elements $\overline{\mathcal{S}}$ of $\overline{\mathcal{A}}$ is equal to $\mathcal{S} \otimes \overline{\mathcal{Z}}$, and the set of skew-symmetric elements $\overline{\mathcal{K}}$ of $\overline{\mathcal{A}}$ is equal to $\mathcal{K} \otimes \overline{\mathcal{Z}}$. 
Observe that $\overline{\mathcal{L}}=\mathcal{L} \otimes \overline{\mathcal{Z}}$ is a Lie skew-ideal of $\overline{\mathcal{A}}$. We now apply the description of an involution on $\overline{\mathcal{A}}$ : there exists a set of matrix units $\left\{e_{i j}\right\}$ in $\overline{\mathcal{A}}$ such that $*$ is either the transpose or the usual symplectic involution relative to $\left\{e_{i j}\right\}$ BMM. Corollary 4.6.13]. The condition that $\operatorname{dim}_{\mathbb{F}} \mathcal{A} \neq 4,16$ implies that $\overline{\mathcal{A}}$ is not isomorphic to $M_{2}(\overline{\mathcal{Z}})$ or $M_{4}(\overline{\mathcal{Z}})$. We may now use Lemmas 3.10 and 3.12 and conclude that $\overline{\mathcal{L}}$ is either 0 , $\overline{\mathcal{Z}}, \overline{\mathcal{K}},[\overline{\mathcal{S}}, \overline{\mathcal{K}}], \overline{\mathcal{S}}, \overline{\mathcal{Z}}+\overline{\mathcal{K}},[\overline{\mathcal{A}}, \overline{\mathcal{A}}]$ or $\overline{\mathcal{A}}$. Note that

$$
\begin{gathered}
0=0 \otimes \overline{\mathcal{Z}}, \quad \overline{\mathcal{Z}}=\mathcal{Z} \otimes \overline{\mathcal{Z}}, \quad \overline{\mathcal{K}}=\mathcal{K} \otimes \overline{\mathcal{Z}}, \quad[\overline{\mathcal{S}}, \overline{\mathcal{K}}]=[\mathcal{S}, \mathcal{K}] \otimes \overline{\mathcal{Z}} \\
\overline{\mathcal{S}}=\mathcal{S} \otimes \overline{\mathcal{Z}}, \overline{\mathcal{Z}}+\overline{\mathcal{K}}=(\mathcal{Z}+\mathcal{K}) \otimes \overline{\mathcal{Z}}, \quad[\overline{\mathcal{A}}, \overline{\mathcal{A}}]=[\mathcal{A}, \mathcal{A}] \otimes \overline{\mathcal{Z}}, \quad \overline{\mathcal{A}}=\mathcal{A} \otimes \overline{\mathcal{Z}}
\end{gathered}
$$

Hence it follows, just as in the proof of Proposition 3.2 that $\mathcal{L}$ is either $0, \mathcal{Z}, \mathcal{K}$, $[\mathcal{S}, \mathcal{K}], \mathcal{S}, \mathcal{Z}+\mathcal{K},[\mathcal{A}, \mathcal{A}]$ or $\mathcal{A}$.

Remark 3.14. Assume the conditions of Theorem 3.13. Consider $\mathcal{L}=[\mathcal{K}, \mathcal{K}]$. Clearly, $\mathcal{L}$ is a Lie skew-ideal of $\mathcal{A}$. Since $\mathcal{L}$ is contained in $\mathcal{K}$, we see from Theorem 3.13 that we have just two possibilities: either $[\mathcal{K}, \mathcal{K}]=0$ or $[\mathcal{K}, \mathcal{K}]=\mathcal{K}$. As one can easily check by passing to $\overline{\mathcal{A}}$, the first possibility is possible only when $\mathcal{A}$ is commutative (or when $\operatorname{dim}_{\mathbb{F}} \mathcal{A}=4$, but this case was excluded by the assumption of Theorem 3.13 . Therefore $[\mathcal{K}, \mathcal{K}]=\mathcal{K}$. This (probably known) fact will be needed in the proof of Theorem 4.10 .

As in the first subsection on Lie ideals, we denote by $\widetilde{\mathcal{A}}$ the central closure of a prime PI algebra $\mathcal{A}$, and by $\widetilde{\mathcal{Z}}$ the field of fractions of $\mathcal{Z}$. By $\widetilde{\mathcal{V}}$ we denote the linear span of $\mathcal{V} \subseteq \mathcal{A}$ over $\widetilde{\mathcal{Z}}$. If $\mathcal{A}$ has an involution of the first kind, then $\widetilde{\mathcal{A}}$ has an involution of the first kind defined by $\left(z^{-1} a\right)^{*}=z^{-1} a^{*}$. Using Theorem 3.13 one can then easily deduce the following corollary.

Corollary 3.15. Let $\mathcal{A}$ be a prime PI algebra with involution of the first kind, and let $\mathcal{L}$ be a Lie skew-ideal of $\mathcal{A}$. If $\operatorname{dim}_{\widetilde{\mathcal{Z}}} \widetilde{\mathcal{A}} \neq 4,16$ and $\operatorname{char}(\mathbb{F}) \neq 2,3$, then $\widetilde{\mathcal{L}}$ is either $0, \widetilde{\mathcal{Z}}, \widetilde{\mathcal{K}},[\widetilde{\mathcal{S}}, \widetilde{\mathcal{K}}], \widetilde{\mathcal{S}}, \widetilde{\mathcal{Z}}+\widetilde{\mathcal{K}},[\widetilde{\mathcal{A}}, \widetilde{\mathcal{A}}]$ or $\widetilde{\mathcal{A}}$.

Let us now consider the simpler case when $*$ is of the second kind, i.e., $*$ is not the identity on $\mathcal{Z}$.

Theorem 3.16. Let $\mathcal{A}$ be a finite dimensional central simple algebra with involution of the second kind, and let $\mathcal{L}$ be a Lie skew-ideal of $\mathcal{A}$. If $\operatorname{char}(\mathbb{F}) \neq 2$, then $\mathcal{L}$ is either $0, \mathcal{Z},[\mathcal{A}, \mathcal{A}]$ or $\mathcal{A}$.

Proof. Since $*$ is of the second kind, there exists $z \in \mathcal{Z}=\mathbb{F}$ such that $w=z-z^{*} \neq 0$. Thus $w$ is nonzero skew-symmetric element in $\mathcal{Z}$. Pick $x \in \mathcal{L}$ and $a \in \mathcal{A}$. We can write $a=s+k$ where $s \in \mathcal{S}$ and $k \in \mathcal{K}$; indeed, we take $s=\frac{a+a^{*}}{2}, k=\frac{a-a^{*}}{2}$. Clearly, $w s \in \mathcal{K}$ and so $[x, w s] \in \mathcal{L}$, and of course also $[x, k] \in \mathcal{L}$. But then $[x, a]=$ $w^{-1}[x, w s]+[x, k] \in \mathcal{L}$. This proves that $[\mathcal{L}, \mathcal{A}] \subseteq \mathcal{L}$; that is, $\mathcal{L}$ is a Lie ideal of $\mathcal{A}$. Now apply Proposition 3.2 .

If $\mathcal{A}$ is a prime PI algebra with involution $*$ of the second kind, then $*$ can be extended to an involution of the second kind of $\widetilde{\mathcal{A}}$ by $\left(z^{-1} a\right)^{*}=z^{*-1} a^{*}$. This readily yields the following corollary.

Corollary 3.17. Let $\mathcal{A}$ be a prime PI algebra with involution of the second kind, and let $\mathcal{L}$ be a Lie skew-ideal of $\mathcal{A}$. If $\operatorname{char}(\mathbb{F}) \neq 2$, then $\widetilde{\mathcal{L}}$ is either $0, \widetilde{\mathcal{Z}},[\widetilde{\mathcal{A}}, \widetilde{\mathcal{A}}]$ or $\widetilde{\mathcal{A}}$. 


\section{Classifying Polynomials and Tracial Nullstellensätze}

The purpose of this section is to classify the polynomials in $\mathbb{F}\langle\bar{X}\rangle$ and in $\mathbb{F}\left\langle\bar{X}, \bar{X}^{*}\right\rangle$ with respect to their values on finite dimensional central simple algebras (with involution), and then as corollaries of these classification results derive what we call "tracial Nullstellensätze".

4.1. Cyclic equivalence. The following notion was introduced in $\mathrm{KS}$.

Definition 4.1. We say that polynomials $f, g$ in $\mathbb{F}\langle\bar{X}\rangle$ (resp. in $\mathbb{F}\left\langle\bar{X}, \bar{X}^{*}\right\rangle$ ) are cyclically equivalent (notation $f \stackrel{\text { cyc }}{\sim} g$ ) if $f-g$ is a sum of commutators in $\mathbb{F}\langle\bar{X}\rangle$ (resp. in $\left.\mathbb{F}\left(\bar{X}, \bar{X}^{*}\right\rangle\right)$.

The next remark shows that cyclic equivalence can be checked easily and that it is "stable" under scalar extensions in the following sense: Given a field extension $\mathbb{F} \subseteq \mathbb{K}$ and $f, g \in \mathbb{F}\langle\bar{X}\rangle$, then $f \stackrel{\text { cyc }}{\sim} g$ in $\mathbb{F}\langle\bar{X}\rangle$ if and only if $f \stackrel{\text { cyc }}{\sim} g$ in $\mathbb{K}\langle\bar{X}\rangle$. We note it holds verbatim for $\mathbb{F}\left\langle\bar{X}, \bar{X}^{*}\right\rangle$ but is stated here only for $\mathbb{F}\langle\bar{X}\rangle$.

\section{Remark 4.2.}

(a) Two words $v, w \in\langle\bar{X}\rangle$ are cyclically equivalent if and only if there are words $v_{1}, v_{2} \in\langle\bar{X}\rangle$ such that $v=v_{1} v_{2}$ and $w=v_{2} v_{1}$.

(b) Two polynomials $f=\sum_{w \in\langle\bar{X}\rangle} a_{w} w$ and $g=\sum_{w \in\langle\bar{X}\rangle} b_{w} w\left(a_{w}, b_{w} \in \mathbb{F}\right)$ are cyclically equivalent if and only if for each $v \in\langle\bar{X}\rangle$,

$$
\sum_{\substack{\text { cyc } \\ w \sim v}} a_{w}=\sum_{\substack{c \text { cyc } \\ w \sim v}} b_{w}
$$

The next two lemmas are simple, but essential for this paper.

Lemma 4.3. Let $f=f\left(X_{1}, \ldots, X_{n}\right) \in \mathbb{F}\langle\bar{X}\rangle$. If $f$ is linear in $X_{n}$, then there exists $g=g\left(X_{1}, \ldots, X_{n-1}\right) \in \mathbb{F}\langle\bar{X}\rangle$ such that $f \stackrel{\text { cyc }}{\sim} g X_{n}$.

Proof. It suffices to treat the case when $f$ is a monomial, that is $f=m X_{n} m^{\prime}$ where $m$ and $m^{\prime}$ are monomials in $X_{1}, \ldots, X_{n-1}$. But then the result follows immediately from the identity $m X_{n} m^{\prime}-m^{\prime} m X_{n}=\left[m X_{n}, m^{\prime}\right]$.

Lemma 4.4. Let $f=f\left(X_{1}, \ldots, X_{n}, X_{1}^{*}, \ldots, X_{n}^{*}\right) \in \mathbb{F}\left\langle\bar{X}, \bar{X}^{*}\right\rangle$. If $f$ is linear in $X_{n}$, then there exist polynomials $g=g\left(X_{1}, \ldots, X_{n-1}, X_{1}^{*}, \ldots, X_{n-1}^{*}\right) \in \mathbb{F}\left\langle\bar{X}, \bar{X}^{*}\right\rangle$ and $g^{\prime}=g^{\prime}\left(X_{1}, \ldots, X_{n-1}, X_{1}^{*}, \ldots, X_{n-1}^{*}\right) \in \mathbb{F}\left\langle\bar{X}, \bar{X}^{*}\right\rangle$ such that $f \stackrel{\text { cyc }}{\sim} g X_{n}+X_{n}^{*} g^{\prime}$.

Proof. The proof is basically the same as the proof of Lemma 4.3 It suffices to consider the case where $f$ is a monomial. If $f=m X_{n} m^{\prime}$ then use $m X_{n} m^{\prime}-m^{\prime} m X_{n}=$ [ $\left.m X_{n}, m^{\prime}\right]$, and if $f=m X_{n}^{*} m^{\prime}$ then use $m X_{n}^{*} m^{\prime}-X_{n}^{*} m^{\prime} m=\left[m, X_{n}^{*} m^{\prime}\right]$.

4.2. Polynomials in $\mathbb{F}\langle\bar{X}\rangle$. Consider first $\mathcal{A}=M_{d}(\mathbb{F})$. Let $f \in \mathbb{F}\langle\bar{X}\rangle$. Theorem 2.3 and Lemma 3.1 imply that span $f(\mathcal{A})$ can be either $0, \mathcal{Z},[\mathcal{A}, \mathcal{A}]$ or $\mathcal{A}$. Each of the four possibilities indeed occurs. Finding polynomials $f$ such that span $f(\mathcal{A})$ is either $[\mathcal{A}, \mathcal{A}]$ or $\mathcal{A}$ is trivial (say, take $X_{1} X_{2}-X_{2} X_{1}$ and $X_{1}$ ). Since $\mathcal{A}$ is a PI algebra, we can find (nonzero) polynomials $f$ such that $\operatorname{span} f(\mathcal{A})=0$. The existence of polynomials $f$ such that $\operatorname{span} f(\mathcal{A})=\mathcal{Z}$ is nontrivial [For, Raz]. These are the so-called central polynomials, i.e., polynomials which are not identities on $\mathcal{A}$ but all their values lie in $\mathcal{Z}$. 
Instead of $M_{d}(\mathbb{F})$ we can consider any finite dimensional central simple algebra $\mathcal{A}$. Again we arrive at four possibilities. Our goal is to determine when each of them occurs.

Theorem 4.5. Let $\mathcal{A}$ be a finite dimensional central simple algebra, let $f \in \mathbb{F}\langle\bar{X}\rangle$, and let us write $\mathcal{L}:=\operatorname{span} f(\mathcal{A})$. If $\operatorname{char}(\mathbb{F})=0$, then exactly one of the following four possibilities holds:

(i) $f$ is an identity of $\mathcal{A}$; in this case $\mathcal{L}=0$;

(ii) $f$ is a central polynomial of $\mathcal{A}$; in this case $\mathcal{L}=\mathcal{Z}$;

(iii) $f$ is not an identity of $\mathcal{A}$, but is cyclically equivalent to an identity of $\mathcal{A}$; in this case $\mathcal{L}=[\mathcal{A}, \mathcal{A}]$

(iv) $f$ is not a central polynomial of $\mathcal{A}$ and is not cyclically equivalent to an identity of $\mathcal{A}$; in this case $\mathcal{L}=\mathcal{A}$.

Proof. Theorem 2.3 and Proposition 3.2 tell us that $\mathcal{L}$ is either $0, \mathcal{Z},[\mathcal{A}, \mathcal{A}]$ or $\mathcal{A}$.

We claim that $\mathcal{Z} \cap[\mathcal{A}, \mathcal{A}]=0$. A standard scalar extension argument shows that it suffices to prove this for the case where $\mathcal{A}=M_{d}(\mathbb{F})$. In this case the desired conclusion follows from the fact that the trace of the identity matrix is $d$ which is, as $\operatorname{char}(\mathbb{F})=0$, different from 0 .

Suppose first that $f$ is cyclically equivalent to an identity. Then $f(\mathcal{A}) \subseteq[\mathcal{A}, \mathcal{A}]$ and hence $\mathcal{L} \subseteq[\mathcal{A}, \mathcal{A}]$. Since $\mathcal{Z} \cap[\mathcal{A}, \mathcal{A}]=0$, there are only two possibilities: either $\mathcal{L}=0$ or $\mathcal{L}=[\mathcal{A}, \mathcal{A}]$. If $f$ itself is an identity, then of course (i) holds. If $f$ is not an identity, then $\mathcal{L} \neq 0$ and so (iii) must hold.

Assume now that $f$ is not cyclically equivalent to an identity. If $f$ is a central polynomial, then (ii) holds. Assume therefore that $f$ is not a central polynomial. We must show that $\mathcal{L}=\mathcal{A}$. Obviously, $\mathcal{L} \neq 0$ and $\mathcal{L} \neq \mathcal{Z}$. We still have to eliminate the possibility that $\mathcal{L}=[\mathcal{A}, \mathcal{A}]$. Assume that this possibility actually occurs, so in particular $f(\mathcal{A}) \subseteq[\mathcal{A}, \mathcal{A}]$. Writing $f$ as a sum of multihomogeneous polynomials, and then arguing as at the beginning of the proof of Theorem 2.3 we see that each of these homogeneous components has the same property that its values lie in $[\mathcal{A}, \mathcal{A}]$. It is obvious that at least one of these summands is not cyclically equivalent to an identity. Thus, there exists a multihomogeneous polynomial, let us call it $h=h\left(X_{1}, \ldots, X_{n}\right)$, which is not cyclically equivalent to an identity and has the property $h(\mathcal{A}) \subseteq[\mathcal{A}, \mathcal{A}]$. We will show that this is impossible by induction on the degree of $h$ with respect to $X_{n}$. Let us denote this degree by $k$. If $k=1$, then we can use Lemma 4.3 to find a polynomial $g=g\left(X_{1}, \ldots, X_{n-1}\right)$ such that $h \stackrel{\text { cyc }}{\sim} g X_{n}$. Consequently, $\left(g X_{n}\right)(\mathcal{A}) \subseteq$ $[\mathcal{A}, \mathcal{A}]$. Pick $a_{1}, \ldots, a_{n-1} \in \mathcal{A}$ and write $w=g\left(a_{1}, \ldots, a_{n-1}\right)$. Then $w x \in[\mathcal{A}, \mathcal{A}]$ for every $x \in \mathcal{A}$, which clearly implies that the same is true for every $x \in \mathcal{A}$. If $w \neq 0$, then because of the simplicity of $\mathcal{A}$ there exist $u_{i}, v_{i} \in \mathcal{A}$ such that $1=\sum_{i} u_{i} w v_{i}$. But then

$$
1=\sum_{i}\left[u_{i}, w v_{i}\right]+w \sum_{i} v_{i} u_{i} \in[\mathcal{A}, \mathcal{A}],
$$

contradicting $\mathcal{Z} \cap[\mathcal{A}, \mathcal{A}]=0$. Thus $w=0$, i.e., $g\left(a_{1}, \ldots, a_{n-1}\right)=0$ for all $a_{i} \in \mathcal{A}$. That is, $g$, and hence also $g X_{n}$, is an identity of $\mathcal{A}$. This contradicts our assumption that $h$ is not cyclically equivalent to an identity. Now let $k>1$ and consider the 
polynomial

$$
\begin{aligned}
h^{\prime}\left(X_{1}, \ldots, X_{n}, X_{n+1}\right) & =h\left(X_{1}, \ldots, X_{n-1}, X_{n}+X_{n+1}\right) \\
& -h\left(X_{1}, \ldots, X_{n-1}, X_{n}\right)-h\left(X_{1}, \ldots, X_{n-1}, X_{n+1}\right) .
\end{aligned}
$$

Obviously the values of $h^{\prime}$ also lie in $[\mathcal{A}, \mathcal{A}]$, and so the same is true for each of multihomogeneous components of $h^{\prime}$. Since the degree in $X_{n}$ of each of these components is smaller than $k$, the induction assumption implies that each of them is cyclically equivalent to an identity. But then $h^{\prime}$ itself is cyclically equivalent to an identity. However, since

$$
h\left(X_{1}, \ldots, X_{n}\right)=\frac{1}{2^{k}-2} h\left(X_{1}, \ldots, X_{n}, X_{n}\right)
$$

it follows that $h$ is also cyclically equivalent to an identity - a contradiction.

Remark 4.6. One easily shows that Theorem 4.5 can be extended to prime PI algebras; the only difference is that, with reference to the notation introduced above, the conclusions in (ii), (iii), and (iv) $\operatorname{read}$ as $\widetilde{\mathcal{L}}=\widetilde{\mathcal{Z}}, \widetilde{\mathcal{L}}=[\widetilde{\mathcal{A}}, \widetilde{\mathcal{A}}]$, and $\widetilde{\mathcal{L}}=\widetilde{\mathcal{A}}$, respectively.

We record the following two easily obtained corollaries related to [KS, Theorem 2.1]. We call them tracial Nullstellensätze; the first one deals with the non-dimensionfree setting and the second one is dimensionfree.

Corollary 4.7. Let $d \geq 2$, let $\operatorname{char}(\mathbb{F})=0$ and let $f=f\left(X_{1}, \ldots, X_{n}\right) \in \mathbb{F}\langle\bar{X}\rangle$. Then $\operatorname{tr}\left(f\left(A_{1}, \ldots, A_{n}\right)\right)=0$ for all $A_{i} \in M_{d}(\mathbb{F})$ if and only if $f$ is cyclically equivalent to an identity of $M_{d}(\mathbb{F})$.

Corollary 4.8. Suppose $\operatorname{char}(\mathbb{F})=0$ and let $f=f\left(X_{1}, \ldots, X_{n}\right) \in \mathbb{F}\langle\bar{X}\rangle$. Then $\operatorname{tr}\left(f\left(A_{1}, \ldots, A_{n}\right)\right)=0$ for all $A_{i} \in M_{d}(\mathbb{F})$ and all $d \geq 2$ if and only if $f \stackrel{\text { cyc }}{\sim} 0$.

4.3. Polynomials in $\mathbb{F}\left\langle\bar{X}, \bar{X}^{*}\right\rangle$. Our aim now is to obtain versions of Theorem 4.5 for polynomials in $\mathbb{F}\left\langle\bar{X}, \bar{X}^{*}\right\rangle$. The situation is easier for involutions of the second kind.

Theorem 4.9. Let $\mathcal{A}$ be a finite dimensional central simple algebra with involution of the second kind, let $f \in \mathbb{F}\left\langle\bar{X}, \bar{X}^{*}\right\rangle$, and let us write $\mathcal{L}:=\operatorname{span} f(\mathcal{A})$. If $\operatorname{char}(\mathbb{F})=0$, then exactly one of the following four possibilities holds:

(i) $f$ is an identity of $\mathcal{A}$; in this case $\mathcal{L}=0$;

(ii) $f$ is a central polynomial of $\mathcal{A}$; in this case $\mathcal{L}=\mathcal{Z}$;

(iii) $f$ is not an identity of $\mathcal{A}$, but is cyclically equivalent to an identity of $\mathcal{A}$; in this case $\mathcal{L}=[\mathcal{A}, \mathcal{A}]$

(iv) $f$ is not a central polynomial of $\mathcal{A}$ and is not cyclically equivalent to an identity of $\mathcal{A}$; in this case $\mathcal{L}=\mathcal{A}$.

Proof. Not only the formulation, also the proof of this theorem is almost literally the same as the proof of Theorem 4.5. Let us therefore just point out a few instances where small changes are necessary. Firstly, one of course has to use Theorem 3.16 (rather than Proposition 3.2) to conclude that $\mathcal{L}$ is $0, \mathcal{Z},[\mathcal{A}, \mathcal{A}]$ or $\mathcal{A}$. Secondly, for the reduction to multihomogeneous polynomials one has to make use only of those scalars that the involution on $\mathbb{F}$ fixes. They also form a field with characteristic 0 , so the same argument works. Thirdly and finally, instead of Lemma 4.3 one has to use Lemma 4.4 
and thereby conclude that $h \stackrel{\text { cyc }}{\sim} g X_{n}+X_{n}^{*} g^{\prime}$, and hence $\left(g X_{n}+X_{n}^{*} g^{\prime}\right)(\mathcal{A}) \subseteq[\mathcal{A}, \mathcal{A}]$. Since the involution is of the second kind this clearly implies that both $\left(g X_{n}\right)(\mathcal{A})$ and $\left(X_{n}^{*} g^{\prime}\right)(\mathcal{A})$ lie in $[\mathcal{A}, \mathcal{A}]$. From this point on the necessary changes are completely obvious.

For an involution of the first kind the situation is somewhat more complicated since Theorem 3.13 yields eight possible classes.

For the ease of exposition we introduce some notation to be used in the next theorem. Let $\mathcal{A}$ be an algebra endowed with a (fixed) involution $* . \quad \operatorname{By} \operatorname{Id}(\mathcal{A})$ we denote the set of all polynomial identities of $\mathcal{A}$ in $\mathbb{F}\left\langle\bar{X}, \bar{X}^{*}\right\rangle$. At this point it seems appropriate to mention that if an algebra satisfies a nontrivial identity in $\mathbb{F}\left\langle\bar{X}, \bar{X}^{*}\right\rangle$, then it also satisfies a nontrivial identity in $\mathbb{F}\langle\bar{X}\rangle$ Ami ; this is why in the $*$-algebra context we confine ourselves to (usual) PI algebras. Next, by $\operatorname{Cen}(\mathcal{A})$ we denote the set of all central polynomials of $\mathcal{A}$ in $\mathbb{F}\langle\bar{X}\rangle$. Note that $\operatorname{Id}(\mathcal{A})$ and $\operatorname{Cen}(\mathcal{A})$ depend on the involution chosen.

Theorem 4.10. Let $\mathcal{A}$ be a finite dimensional central simple algebra with involution of the first kind, let $f \in \mathbb{F}\left\langle\bar{X}, \bar{X}^{*}\right\rangle$, and let us write $\mathcal{L}:=\operatorname{span} f(\mathcal{A})$. If $\operatorname{dim}_{\mathbb{F}} \mathcal{A} \neq 1,4,16$ and $\operatorname{char}(\mathbb{F})=0$, then exactly one of the following eight possibilities holds:

(i) $f \in \operatorname{Id}(\mathcal{A})$; in this case $\mathcal{L}=0$;

(ii) $f \in \operatorname{Cen}(\mathcal{A})$; in this case $\mathcal{L}=\mathcal{Z}$;

(iii) $f \in \operatorname{Skew} \mathbb{F}\left\langle\bar{X}, \bar{X}^{*}\right\rangle+\operatorname{Id}(\mathcal{A})$ and $f \notin \operatorname{Id}(\mathcal{A})$; in this case $\mathcal{L}=\mathcal{K}$;

(iv) $f \in \operatorname{Skew} \mathbb{F}\left\langle\bar{X}, \bar{X}^{*}\right\rangle+\operatorname{Cen}(\mathcal{A})$ and $f \notin \operatorname{Cen}(\mathcal{A})$; in this case $\mathcal{L}=\mathcal{Z}+\mathcal{K}$;

(v) $f \in \operatorname{Sym} \mathbb{F}\left\langle\bar{X}, \bar{X}^{*}\right\rangle+\operatorname{Id}(\mathcal{A}), f \notin \operatorname{Id}(\mathcal{A})$ and $f$ is cyclically equivalent to an element of $\operatorname{Id}(\mathcal{A})$; in this case $\mathcal{L}=[\mathcal{S}, \mathcal{K}]$;

(vi) $f \in \operatorname{Sym} \mathbb{F}\left\langle\bar{X}, \bar{X}^{*}\right\rangle+\operatorname{Id}(\mathcal{A}), f \notin \operatorname{Cen}(\mathcal{A})$ and $f$ is not cyclically equivalent to an element of $\operatorname{Id}(\mathcal{A})$; in this case $\mathcal{L}=\mathcal{S}$;

(vii) $f \notin \operatorname{Sym} \mathbb{F}\left\langle\bar{X}, \bar{X}^{*}\right\rangle+\operatorname{Id}(\mathcal{A}), f \notin \operatorname{Skew} \mathbb{F}\left\langle\bar{X}, \bar{X}^{*}\right\rangle+\operatorname{Id}(\mathcal{A})$, and $f+f^{*}$ is cyclically equivalent to an element of $\operatorname{Id}(\mathcal{A})$; in this case $\mathcal{L}=[\mathcal{A}, \mathcal{A}]$;

(viii) $f \notin \operatorname{Sym} \mathbb{F}\left\langle\bar{X}, \bar{X}^{*}\right\rangle+\operatorname{Id}(\mathcal{A}), f \notin \operatorname{Skew} \mathbb{F}\left\langle\bar{X}, \bar{X}^{*}\right\rangle+\operatorname{Id}(\mathcal{A}), f \notin \operatorname{Skew} \mathbb{F}\left\langle\bar{X}, \bar{X}^{*}\right\rangle+$ $\operatorname{Cen}(\mathcal{A})$ and $f+f^{*}$ is not cyclically equivalent to an element of $\operatorname{Id}(\mathcal{A})$; in this case $\mathcal{L}=\mathcal{A}$.

Proof. We start by remarking that $\mathcal{L}$ is a Lie skew-ideal of $\mathcal{A}$ by Theorem 2.5. Therefore $\mathcal{L}$ is either $0, \mathcal{Z}, \mathcal{K},[\mathcal{S}, \mathcal{K}], \mathcal{S}, \mathcal{Z}+\mathcal{K},[\mathcal{A}, \mathcal{A}]$ or $\mathcal{A}$ by Theorem 3.13 .

We divide the proof into two parts, (a) and (b), depending on whether or not $f+f^{*}$ is cyclically equivalent to an element of $\operatorname{Id}(\mathcal{A})$.

(a) Assume that $f+f^{*}$ is cyclically equivalent to an identity. Then $f=\frac{f+f^{*}}{2}+\frac{f-f^{*}}{2}$ is a sum of an identity, commutators, and a skew-symmetric polynomial, and hence $f(\mathcal{A}) \subseteq[\mathcal{A}, \mathcal{A}]+\mathcal{K} \subseteq[\mathcal{A}, \mathcal{A}]+\mathcal{K}$. In Remark 3.14 we have noticed that $\mathcal{K}=[\mathcal{K}, \mathcal{K}]$. This forces $f(\mathcal{A}) \subseteq[\mathcal{A}, \mathcal{A}]$, and consequently $\mathcal{L} \subseteq[\mathcal{A}, \mathcal{A}]$.

Recall from the proof of Theorem 4.5 that $\mathcal{Z} \cap[\mathcal{A}, \mathcal{A}]=0$. Therefore $\mathcal{L}$ is neither $\mathcal{Z}, \mathcal{Z}+\mathcal{K}, \mathcal{S}$ nor $\mathcal{A}$. Thus $\mathcal{L} \in\{0, \mathcal{K},[\mathcal{S}, \mathcal{K}],[\mathcal{A}, \mathcal{A}]\}$. If $f$ itself is an identity, then of course (i) holds. Now suppose $f$ is not an identity. If $f \in \operatorname{Skew} \mathbb{F}\left\langle\bar{X}, \bar{X}^{*}\right\rangle+\operatorname{Id}(\mathcal{A})$, then (iii) holds. If $f \in \operatorname{Sym} \mathbb{F}\left\langle\bar{X}, \bar{X}^{*}\right\rangle+\operatorname{Id}(\mathcal{A})$, then (v) holds. Otherwise (vii) holds. Let us also point out that $f$ cannot belong to Skew $\mathbb{F}\left\langle\bar{X}, \bar{X}^{*}\right\rangle+\operatorname{Cen}(\mathcal{A})$ if (vii) occurs.

(b) Now assume that $f+f^{*}$ is not cyclically equivalent to an identity. Let us first show that $\mathcal{L} \nsubseteq[\mathcal{A}, \mathcal{A}]$. Suppose this is not true, that is, suppose $f(\mathcal{A}) \subseteq$ 
$[\mathcal{A}, \mathcal{A}]$. As a skew-symmetric polynomial, $f-f^{*}$ automatically satisfies $\left(f-f^{*}\right)(\mathcal{A}) \subseteq$ $\mathcal{K} \subseteq[\mathcal{A}, \mathcal{A}]$ by Remark 3.14. But then $s=f+f^{*}=2 f-\left(f-f^{*}\right)$ has the same property, i.e., $s(\mathcal{A}) \subseteq[\mathcal{A}, \mathcal{A}]$. Suppose that $s$ is linear in $X_{n}$. Then Lemma 4.4 tells us that there exist $g=g\left(X_{1}, \ldots, X_{n-1}, X_{1}^{*}, \ldots, X_{n-1}^{*}\right) \in \mathbb{F}\left\langle\bar{X}, \bar{X}^{*}\right\rangle$ and $g^{\prime}=g^{\prime}\left(X_{1}, \ldots, X_{n-1}, X_{1}^{*}, \ldots, X_{n-1}^{*}\right) \in \mathbb{F}\left\langle\bar{X}, \bar{X}^{*}\right\rangle$ such that $s \stackrel{\text { cyc }}{\sim} g X_{n}+X_{n}^{*} g^{\prime}$. It is clear that then $\left(g X_{n}+X_{n}^{*} g^{\prime}\right)(\mathcal{A}) \subseteq[\mathcal{A}, \mathcal{A}]$. Pick $a_{1}, \ldots, a_{n-1} \in \mathcal{A}$ and set $b=$ $g\left(a_{1}, \ldots, a_{n-1}, a_{1}^{*}, \ldots, a_{n-1}^{*}\right), c=g^{\prime}\left(a_{1}, \ldots, a_{n-1}, a_{1}^{*}, \ldots, a_{n-1}^{*}\right)$. Then $b x+x^{*} c \in$ $[\mathcal{A}, \mathcal{A}]$ for all $x \in \mathcal{A}$, and hence also for all $x \in \mathcal{A}$. Consequently,

$$
\left(b+c^{*}\right) x=\left(b x+x^{*} c\right)+\left(c^{*} x-x^{*} c\right) \in[\mathcal{A}, \mathcal{A}]+\mathcal{K}=[\mathcal{A}, \mathcal{A}] .
$$

Thus $w \mathcal{A} \subseteq[\mathcal{A}, \mathcal{A}]$ where $w=b+c^{*}$. As in the proof of Theorem 4.5 we see that this yields $w=0$, i.e.,

$$
g\left(a_{1}, \ldots, a_{n-1}, a_{1}^{*}, \ldots, a_{n-1}^{*}\right)+g^{\prime}\left(a_{1}, \ldots, a_{n-1}, a_{1}^{*}, \ldots, a_{n-1}^{*}\right)^{*}=0 .
$$

Since the $a_{i}$ 's are arbitrary elements in $\mathcal{A}$, this means that $g+g^{\prime *} \in \operatorname{Id}(\mathcal{A})$. Thus

$$
s \stackrel{\text { cyc }}{\sim} g X_{n}+X_{n}^{*} g^{\prime}=\left(-h^{*} X_{n}+X_{n}^{*} g^{\prime}\right)+\left(g+g^{*}\right) X_{n} \in \operatorname{Skew} \mathbb{F}\left\langle\bar{X}, \bar{X}^{*}\right\rangle+\operatorname{Id}(\mathcal{A}) .
$$

Since $s=f+f^{*} \in \operatorname{Sym} \mathbb{F}\left\langle\bar{X}, \bar{X}^{*}\right\rangle$ and since both Skew $\mathbb{F}\left\langle\bar{X}, \bar{X}^{*}\right\rangle$ and $\operatorname{Id}(\mathcal{A})$ are invariant under $*$, we now arrive at the contradiction that $s$ is cyclically equivalent to an element in $\operatorname{Id}(\mathcal{A})$. Recall that this was derived under the assumption that $s$ is linear in $X_{n}$. The general case can be reduced to this one in the same way as in the proof of Theorem 4.5. Therefore we have indeed $\mathcal{L} \nsubseteq[\mathcal{A}, \mathcal{A}]$.

We now know that $\mathcal{L} \in\{\mathcal{Z}, \mathcal{S}, \mathcal{Z}+\mathcal{K}, \mathcal{A}\}$. If $f \in \operatorname{Cen}(\mathcal{A})$, then (ii) holds. Suppose now that $f$ is not a central polynomial. If $f \in \operatorname{Skew} \mathbb{F}\left\langle\bar{X}, \bar{X}^{*}\right\rangle+\operatorname{Cen}(\mathcal{A})$, then (iv) holds. If $f \in \operatorname{Sym} \mathbb{F}\left\langle\bar{X}, \bar{X}^{*}\right\rangle+\operatorname{Id}(\mathcal{A})$, then (vi) must hold. Otherwise we have (viii).

Due to the construction of the cases (i) - (viii) it is clear that they are exhaustive and mutually exclusive.

Remark 4.11. With the presence of ${ }^{\sim}$, both Theorems 4.9 and 4.10 can be easily extended to prime PI algebras (cf. Remark 4.6).

We are now in a position to give the tracial Nullstellensätze for free $*$-algebras:

Corollary 4.12. Let $d \neq 1,2,4$, let $\operatorname{char}(\mathbb{F})=0$ and let $f \in \mathbb{F}\left\langle\bar{X}, \bar{X}^{*}\right\rangle$ be a polynomial in $n$ variables. Fix an involution $*$ on $M_{d}(\mathbb{F})$. If it is of the first kind, assume that $f \in \operatorname{Sym} \mathbb{F}\left\langle\bar{X}, \bar{X}^{*}\right\rangle$. Then $\operatorname{tr}\left(f\left(A_{1}, \ldots, A_{n}, A_{1}^{*}, \ldots, A_{n}^{*}\right)\right)=0$ for all $A_{i} \in M_{d}(\mathbb{F})$ if and only if $f$ is cyclically equivalent to an identity of $M_{d}(\mathbb{F})$.

Corollary 4.13 (cf. Theorem 2.1 in $[\mathrm{KS}]$ ). Let $\operatorname{char}(\mathbb{F})=0$ and let $f \in \mathbb{F}\left\langle\bar{X}, \bar{X}^{*}\right\rangle$ be a polynomial in $n$ variables. Fix an involution $*$ on $M_{d}(\mathbb{F})$. If it is of the first kind, assume that $f \in \operatorname{Sym} \mathbb{F}\left\langle\bar{X}, \bar{X}^{*}\right\rangle$. Then $\operatorname{tr}\left(f\left(A_{1}, \ldots, A_{n}, A_{1}^{*}, \ldots, A_{n}^{*}\right)\right)=0$ for all $A_{i} \in M_{d}(\mathbb{F})$ and all $d \geq 2$ if and only if $f \stackrel{\text { cyc }}{\sim} 0$.

Remark 4.14. The results given in this subsection can be easily extended to free algebras with involution generated by symmetric (or skew-symmetric) variables. 


\section{Algebra of generic matrices}

In this section we interpret some of our main results in the algebra of generic matrices, cf. [Pro1, Chapter III] or [Row, \$1.3]. As is often the case, verifying a condition on values of a polynomial on $d \times d$ matrices is conveniently done in the algebra of generic matrices. Here we discuss "having zero trace" and present appropriate versions of Corollaries 4.7 (in Theorem 5.1) and 4.12 (in Theorem 5.2.

Somewhat related is the result of Amitsur and Rowen [AR, where they show that in central simple algebras an element is a sum of (two) commutators if and only if its reduced trace is zero; see also $\underline{\mathrm{AR}}$, Appendix to 3] and $[\mathrm{RR}$.

5.1. Algebra of generic matrices (without involution). Let $\zeta:=\left(\zeta_{i j}^{(\ell)} \mid 1 \leq\right.$ $i, j \leq d, \ell \in \mathbb{N}$ ) denote commuting variables and form the polynomial algebra $\mathbb{F}[\zeta]$. Then the algebra of generic $d \times d$ matrices $\mathrm{GM}_{d}(\mathbb{F})$ is the subalgebra of $M_{d}(\mathbb{F}[\zeta])$ generated by the $d \times d$ matrices $Y_{\ell}:=\left[\zeta_{i j}^{(\ell)}\right]_{1 \leq i, j \leq d}$, where $\ell \in \mathbb{N}$. Each $Y_{\ell}$ is called a generic matrix. Furthermore, $\mathrm{GM}_{d}(\mathbb{F}, n)$ is used to denote the subalgebra generated by the $n$ generic matrices $Y_{1}, \ldots, Y_{n}$. The algebra of generic $d \times d$ matrices is a PI algebra and a domain. Moreover, $\mathrm{GM}_{d}(\mathbb{F})$ is isomorphic to $\mathbb{F}\langle\bar{X}\rangle / \mathrm{Id}_{d}$, where $\operatorname{Id}_{d}$ is the ideal of all polynomial identities of $d \times d$ matrices. For details and proofs we refer the reader to [Pro1, Chapters I and III] or [Row, §1.3] Our approach to generic matrices is purely algebraic. A representation-theoretic viewpoint with a more geometric flavor can be found in Pro2.

$\mathrm{GM}_{d}(\mathbb{F})$ enjoys the following property: any algebra homomorphism

$$
\mathrm{ev}_{a}: \mathbb{F}[\zeta] \rightarrow \mathbb{F}, \quad p \mapsto p(a)
$$

"lifts" to a homomorphism of algebras $\mathrm{GM}_{d}(\mathbb{F}) \rightarrow M_{d}(\mathbb{F})$ by entrywise evaluation. The image of an element $f \in \mathrm{GM}_{d}(\mathbb{F})$ under this map will be denoted simply by $f(a)$.

Theorem 5.1 (Tracial Nullstellensatz for generic matrices). Suppose char $(\mathbb{F})=0$ and let $f \in \mathrm{GM}_{d}(\mathbb{F})$. Then the following are equivalent:

(i) $f$ is a sum of commutators in $\mathrm{GM}_{d}(\mathbb{F})$;

(ii) $\operatorname{tr}(f)=0$;

(iii) $\operatorname{tr}(f(a))=0$ for all $a \in M_{d}(\mathbb{F})^{\mathbb{N}}$;

(iv) $f(a)$ is a sum of commutators in $M_{d}(\mathbb{F})$ for all $a \in M_{d}(\mathbb{F})^{\mathbb{N}}$.

Proof. The equivalences (ii) $\Leftrightarrow$ (iii) and (iii) $\Leftrightarrow$ (iv) are obvious as is the implication (i) $\Rightarrow$ (iii). For the proof of (iii) $\Rightarrow$ (i) let $F=F\left(X_{1}, \ldots, X_{n}\right) \in \mathbb{F}\langle\bar{X}\rangle$ denote a preimage of $f$ under the homomorphism $\mathbb{F}\langle\bar{X}\rangle \rightarrow \mathrm{GM}_{d}(\mathbb{F})$. Then $f(a)=F\left(Y_{1}(a), \ldots, Y_{n}(a)\right)$. As $a$ runs through all of $M_{d}(\mathbb{F})^{\mathbb{N}},\left(Y_{1}(a), \ldots, Y_{n}(a)\right)$ sweeps through all $n$-tuples of $d \times d$ matrices over $\mathbb{F}$. By assumption, $\operatorname{tr}\left(F\left(A_{1}, \ldots, A_{n}\right)\right)=0$ for all $A_{i} \in M_{d}(\mathbb{F})$. Hence Corollary 4.7 implies that $F$ is cyclically equivalent to an identity of $M_{d}(\mathbb{F})$. Thus $f$ is a sum of commutators.

It is clear that a similar statement holds for $f \in \mathrm{GM}_{d}(\mathbb{F}, n)$.

5.2. Algebra of generic matrices with involution. Like in the classical construction of the algebra of generic matrices, it is possible to construct the algebra of generic matrices with involution, cf. [Pro2, §20] or [PS, §II]. To each type of involution (orthogonal, symplectic and unitary) an algebra of generic matrices with involution 
can be associated. We proceed to describe details. From now we assume that $\mathbb{F}$ is a field of characteristic 0 with an involution.

Let $\mathbb{F}\left\langle\bar{X}, \bar{X}^{*}\right\rangle$ be the free $*$-algebra over $\mathbb{F}$. For $\mathrm{J} \in$ \{symplectic, orthogonal, unitary $\}$ and an involution of type $\mathrm{J}_{\text {, let }} \operatorname{Id}_{\mathrm{J}, d} \subseteq \mathbb{F}\left\langle\bar{X}, \bar{X}^{*}\right\rangle$ denote the ideal of all identities satisfied by degree $d$ central simple algebras with involution of type J [Row, §2] (a word of caution: the notation has changed from Section 4.3 in order to emphasize the dependence on the (type of) involution). That is, $f=f\left(X_{1}, \ldots, X_{k}, X_{1}^{*}, \ldots, X_{k}^{*}\right) \in$ $\mathbb{F}\left\langle\bar{X}, \bar{X}^{*}\right\rangle$ is an element of $\operatorname{Id}_{\mathrm{J}, d}$ if and only if for every central simple algebra $\mathcal{A}$ with involution of type $\mathrm{J}$ of degree $d$ and every $a_{1}, \ldots, a_{k} \in \mathcal{A}$,

$$
f\left(a_{1}, \ldots, a_{k}, a_{1}^{*}, \ldots, a_{k}^{*}\right)=0 .
$$

Then $\mathrm{GM}_{d}(\mathbb{F}, \mathrm{J}):=\mathbb{F}\left\langle\bar{X}, \bar{X}^{*}\right\rangle / \mathrm{Id}_{\mathrm{J}, d}$ is the algebra of generic $d \times d$ matrices with involution of type $\mathrm{J}$.

Let $\zeta, Y_{\ell}$ be as above. The involution on $\mathbb{F}$ is extended to $\mathbb{F}[\zeta]$ by fixing the $\zeta_{i j}^{(\ell)}$ pointwise.

(1) If $\mathrm{J}=$ orthogonal or $\mathrm{J}=$ unitary, then $\mathrm{GM}_{d}(\mathbb{F}, \mathrm{J})$ is canonically isomorphic to the (unital) $\mathbb{F}$-subalgebra of $M_{d}(\mathbb{F}[\zeta])$ generated by the $Y_{\ell}$ and their transposes.

(2) If $\mathrm{J}=$ symplectic, then $d$ is even, say $d=2 d_{0}$, and the (unital) $\mathbb{F}$-subalgebra of $M_{2 d_{0}}(\mathbb{F}[\zeta])$ generated by the $Y_{\ell}$ and their images under the usual symplectic involution is (canonically) isomorphic to $\mathrm{GM}_{2 d_{0}}(\mathbb{F}, \mathrm{J})$.

Let $\mathbb{F}_{0}$ denote the set of all symmetric elements of $\mathbb{F}$. Then every $*$-algebra homomorphism $\mathbb{F}[\zeta] \rightarrow \mathbb{F}$ is described by a point $a \in M_{d}\left(\mathbb{F}_{0}\right)^{\mathbb{N}}$ and given by the images of $\zeta_{i j}^{(\ell)}$. Hence it induces a $*$-algebra (evaluation) homomorphism $\mathrm{GM}_{d}(\mathbb{F}, \mathrm{J}) \rightarrow M_{d}(\mathbb{F})$ denoted by $g \mapsto g\left(a, a^{*}\right)$. If $G \in \mathbb{F}\left\langle\bar{X}, \bar{X}^{*}\right\rangle$ is a polynomial whose coset in $\operatorname{GM}_{d}(\mathbb{F}, J)$ is represented by $g$, then $g\left(a, a^{*}\right)$ equals $G\left(a, a^{*}\right)$, the evaluation of $G$ at the tuple of $d \times d$ matrices $a$. This means that, as before, any $*$-algebra homomorphism

$$
\mathrm{ev}_{a}: \mathbb{F}[\zeta] \rightarrow \mathbb{F}, \quad p \mapsto p\left(a, a^{*}\right)
$$

lifts to a $*$-homomorphism $\mathrm{GM}_{d}(\mathbb{F}, J) \rightarrow M_{d}(\mathbb{F})$ by entrywise evaluation.

Theorem 5.2 (Tracial Nullstellensatz for generic matrices with involution). Suppose $\operatorname{char}(\mathbb{F})=0$, fix a type $\mathrm{J}$ and let $f \in \mathrm{GM}_{d}(\mathbb{F}, \mathrm{J})$. Write $\mathbb{F}_{0}$ for the set of symmetric elements of $\mathbb{F}$. If $\mathrm{J} \neq$ unitary, assume moreover that $f=f^{*}$. Then the following are equivalent:

(i) $f$ is a sum of commutators in $\mathrm{GM}_{d}(\mathbb{F}, \mathrm{J})$;

(ii) $\operatorname{tr}(f)=0$;

(iii) $\operatorname{tr}\left(f\left(a, a^{*}\right)\right)=0$ for all $a \in M_{d}\left(\mathbb{F}_{0}\right)^{\mathbb{N}}$;

(iv) $f\left(a, a^{*}\right)$ is a sum of commutators in $M_{d}(\mathbb{F})$ for all $a \in M_{d}\left(\mathbb{F}_{0}\right)^{\mathbb{N}}$.

The proof is essentially the same as that of Theorem 5.1 and is therefore omitted.

\section{References}

[AM] A. A. Albert, B. Muckenhoupt, On matrices of trace zero, Michigan Math. J. 4 (1957) 1-3.

[Ami] S. A. Amitsur, Identities in rings with involutions, Israel J. Math. 7 (1969) 63-68.

[AR] S. A. Amitsur, L. H. Rowen, Elements of reduced trace 0, Israel J. Math. 87 (1994) 161179 . 
[BMM $]$ K.I. Beidar, W.S. Martindale 3rd, A.V. Mikhalev, Rings with generalized identities, Marcel Dekker, Inc., 1996.

[BCM] M. Brešar, M. A. Chebotar, W.S. Martindale, Functional identities, Birkhäuser Verlag, 2007.

[BKS] M. Brešar, E. Kissin, V. Shulman, Lie ideals: from pure algebra to $\mathrm{C}^{*}$-algebras, J. Reine Angew. Math. 623 (2008) 73-121.

[Coh] P. M. Cohn, Skew fields. Theory of general division rings, Cambridge University Press, 1995.

[dOHMP] M. C. de Olivera, J. W. Helton, S. A. McCullough, M. Putinar, Engineering Systems and Free Semi-Algebraic Geometry, Emerging Applications of Algebraic Geometry, 17-62, IMA Vol. Math. Appl. 149, Springer, 2008.

[For] E. Formanek, Central polynomials for matrix rings, J. Algebra 23 (1972) 129-132.

[Hel] J. W. Helton, "Positive" noncommutative polynomials are sums of squares, Ann. of Math. (2) 156 (2002) 675-694.

[Her1] I. N. Herstein, Certain submodules of simple rings with involution, Duke Math. J. 24 (1957) 357-364.

[Her2] I. N. Herstein, Topics in ring theory, The University of Chicago Press, 1969.

[KS] I. Klep, M. Schweighofer, Connes' embedding conjecture and sums of hermitian squares, Adv. Math. 217 (2008) 1816-1837.

[KU] I. Klep, T. Unger, The Procesi-Schacher conjecture and Hilbert's 17th problem for algebras with involution, preprint http://arxiv.org/abs/0810.5254

[KMRT] M.-A. Knus, A. Merkurjev, M. Rost, J.-P. Tignol, The book of involutions, American Mathematical Society, 1998.

[Lan] C. Lanski, Unitary invariance in algebraic algebras, Trans. Amer. Math. Soc. 245 (1978) 139-146.

[Mon] S. Montgomery, Invariant subgroups in rings with involution, Can. J. Math. 30 (1978) 350-357.

[Pro1] C. Procesi, Rings with polynomial identities, Marcel Dekker, Inc., 1973.

[Pro2] C. Procesi, The invariant theory of $n \times n$ matrices, Advances in Math. 19 (1976) 306-381.

[PS] C. Procesi, M. Schacher, A non-commutative real Nullstellensatz and Hilbert's 17th problem, Ann. of Math. (2) 104 (1976) 395-406.

[Raz] Y.P. Razmyslov, A certain problem of Kaplansky, Izv. Acad. Nauk SSSR Ser. Mat. 37 (1973) 67-74.

[RR] M. Rosset, S. Rosset, Elements of trace zero that are not commutators, Comm. Algebra 28 (2000) 3059-3072.

[Row] L. H. Rowen, Polynomial identities in ring theory, Academic Press, 1980

[Sho] K. Shoda, Einige Sätze über Matrizen, Japanese J. Math. 13 (1936) 361-365.

Faculty of Mathematics and Physics, University of Ljubluana, and, Faculty of Natural Sciences and Mathematics, University of Maribor, Slovenia

E-mail address: matej.bresar@fmf.uni-lj.si

Faculty of Mathematics and Physics, University of Ljubluana, and, Faculty of Natural Sciences and Mathematics, University of Maribor, Slovenia

E-mail address: igor.klep@fmf.uni-lj.si 